\title{
STANDARD STATIC FINSLER SPACETIMES
}

\author{
ERASMO CAPONIO AND GIUSEPPE STANCARONE
}

\begin{abstract}
We introduce the notion of a standard static Finsler spacetime $\mathbb{R} \times M$ where the base $M$ is a Finsler manifold. We prove some results which connect causality with the Finslerian geometry of the base extending analogous ones for static and stationary Lorentzian spacetimes.
\end{abstract}

\section{INTRODUCTION}

Recent years have seen a growing interest in Finsler spacetimes. Indeed, Finslerian modifications of general relativity have been proposed and Finsler spacetimes have been considered as possible backgrounds for the Standard Model of particles and for quantum gravity with Lorentz-symmetry violating fields, see, e.g., [39], [40], [49], [50], [6], [48], [22], [29], [42], [25], [26], [47], [45], [27]. Moreover, several authors have started to investigate the geometric and the causal structure of Finsler spacetimes, see, e.g., [38], [28], [39], [20], [24], [34], [35], [1]. Different definitions of a Finsler spacetime metric have been proposed in the last cited papers but, with the exception of [28], they do not include a somehow natural generalization to the Finsler realm of the notions of standard static and standard stationary spacetimes. This is related to the fact that such classes of spacetime are defined on product manifolds $\tilde{M}=\mathbb{R} \times M$ where $M$ is identifiable with a spacelike hypersurface of $\tilde{M}$. So a Lorentzian Finsler structure on $\tilde{M}$ inducing a classical Finsler structure on $M$ cannot be smoothly extended (where smooth here means at least $C^{2}$ ) to vectors which project trivially on $T M$, due to the lack of regularity of a Finsler function on the zero section.

Motivated by the above problem, we define a Finsler spacetime as follows. Let $\tilde{M}$ be a $(n+1)$-dimensional smooth paracompact connected manifold, $n \geq 1$. Let us denote by $T \tilde{M}$ its tangent bundle and by 0 the zero section. Let $\mathcal{T} \subset T \tilde{M}$ be a smooth real line vector bundle on $\tilde{M}$ and $\mathcal{T}_{p}$ the fibre of $\mathcal{T}$ over $p \in \tilde{M}$. Let $\pi: T \tilde{M} \backslash \mathcal{T} \rightarrow \tilde{M}$ be the restriction of the canonical projection, $\tilde{\pi}: T \tilde{M} \rightarrow \tilde{M}$, to $T \tilde{M} \backslash \mathcal{T}$ and let $\pi^{*}\left(T^{*} \tilde{M}\right)$ the pulled-back cotangent bundle over $T \tilde{M} \backslash \mathcal{T}$. Let us consider the tensor bundle $\pi^{*}\left(T^{*} \tilde{M}\right) \otimes \pi^{*}\left(T^{*} \tilde{M}\right)$ over $T \tilde{M} \backslash \mathcal{T}$ and a section $\tilde{g}: v \in$ $T \tilde{M} \backslash \mathcal{T} \mapsto \tilde{g}_{v} \in T_{\pi(v)}^{*} \tilde{M} \otimes T_{\pi(v)}^{*} \tilde{M}$. We say that $\tilde{g}$ is symmetric if $\tilde{g}_{v}$ is symmetric for all $v \in T \tilde{M} \backslash \mathcal{T}$. Analogously, $\tilde{g}$ is said non-degenerate if $\tilde{g}_{v}$ is non-degenerate for each $v \in T \tilde{M} \backslash \mathcal{T}$ and its index will be the common index of the symmetric bilinear forms $\tilde{g}_{v}$; moreover, $\tilde{g}$ will be said homogeneous if, for all $\lambda>0$ and $v \in T \tilde{M} \backslash \mathcal{T}, \tilde{g}_{\lambda v}=\tilde{g}_{v}$. Finally, if the above conditions on $\tilde{g}$ are satisfied, we say that $\tilde{g}$ is the vertical Hessian of a (quadratic) Finsler function if there exists a function

2010 Mathematics Subject Classification. 53B30, 53B40, 53C50, 53C60.

Key words and phrases. Finsler spacetime, static spacetime, causality. 
$L: T \tilde{M} \rightarrow \mathbb{R}$ such that in natural coordinates $\left(x^{0}, x^{1}, \ldots, x^{n}, v^{0}, v^{1}, \ldots, v^{n}\right)$ of $T \tilde{M}$, $\frac{1}{2} \frac{\partial^{2} L}{\partial v^{2} \partial v^{j}}(v)=\tilde{g}_{v}$, for all $v \in T \tilde{M} \backslash \mathcal{T}$.

Definition 1.1. A Finsler spacetime $(\tilde{M}, L)$ is a smooth $(n+1)$-dimensional manifold $\tilde{M}, n \geq 1$, endowed with a smooth, symmetric, homogeneous, non-degenerate of index 1 section $\tilde{g}$ of the tensor bundle $\pi^{*}\left(T^{*} \tilde{M}\right) \otimes \pi^{*}\left(T^{*} \tilde{M}\right)$ over $T \tilde{M} \backslash \mathcal{T}$, which is the vertical Hessian of a Finsler function $L$ and such that $\tilde{g}_{w}(w, w)<0$ for each $w$ in a punctured conic neighbourhood of $\mathcal{T}_{p}$ in $T_{p} \tilde{M} \backslash\{0\}$ and for all $p \in \tilde{M}$.

Remark 1.2. Clearly, the Finsler function in the above definition must be fiberwise positively homogeneous of degree 2 and, so, it is, except for the possible lack of twice differentiability along $\mathcal{T}$, an indefinite Finsler (also called Lorentz-Finsler or simply Finsler) function as, e.g., in [7, 34, 38]. We could allow more generality by not prescribing the existence of such a function. This is a quite popular approach to Finsler geometry: see, e.g., [4, 33], and the references therein, where such structures are called generalized metrics (although in [33] they are sections of the tensor bundle $\tilde{\pi}^{*}\left(T^{*} \tilde{M}\right) \otimes \tilde{\pi}^{*}\left(T^{*} \tilde{M}\right)$ with base the whole $\left.T \tilde{M}\right)$. By homogeneity, it is easy to prove that a generalized homogeneous metric is the vertical Hessian of a smooth Finsler function on $T \tilde{M} \backslash \mathcal{T}$ if and only if its Cartan tensor is totally symmetric, i.e. $\frac{\partial \tilde{g}_{i j}}{\partial v^{k}}(v)=\frac{\partial \tilde{g}_{i k}}{\partial v^{j}}(v)$ for all $v \in T \tilde{M} \backslash \mathcal{T}$ (see, e.g., [4, Theorem 3.4.2.1]).

Anyway, there are some arguments against the definition of a Finsler spacetime without a Finsler function which will be considered in Remark 2.11.

Remark 1.3. The requirement about the sign of $\tilde{g}_{w}(w, w)$ for $w$ in a neighbourhood of $\mathcal{T}$ could be weakened by allowing the existence of an open subset $\tilde{M}_{l} \subset \tilde{M}$ where the reverse inequality holds for any $w$ in a punctured neighbourhood of $\mathcal{T}_{p}$, for all $p \in \tilde{M}_{l}$ (and then, eventually, the existence of a "critical region" where, in each punctured neighbourhood of $\mathcal{T}_{p}$, there exist vectors $w_{1}$ and $w_{2}$ such that $\tilde{g}_{w_{1}}\left(w_{1}, w_{1}\right)<0$ and $\left.\tilde{g}_{w_{2}}\left(w_{2}, w_{2}\right)>0\right)$. This, for example, would be the case for a Finslerian modification of a class of spacetime called SSTK splitting and studied in [15] (see last section).

Remark 1.4. Whenever $\mathcal{T}$ is trivial and $\tilde{g}$ can be smoothly extended to $\mathcal{T} \backslash 0$, we recover the definition of a time-orientable Finsler spacetime in [34] by choosing a no-where zero continuous section $T$ such that $T_{p} \in \mathcal{T}$, for each $p \in \tilde{M}$.

Remark 1.5. Definition 1.1 of a Finsler spacetime is a slight generalization of the ones appearing in $[7,38,34]$ as it takes into account the problem of defining particular Finslerian warped products. It is less general than one given in [28] where $L$ is a.e. smooth on $T \tilde{M}$. We point out that all these definitions are purely kinematical in the sense that they do not take into account the problem of determining physical reasonable Finslerian field equations (see the discussion of this problem in [49]).

Definition 1.6. A vector $w \in T \tilde{M}$ is called timelike if either $w \in \mathcal{T} \backslash 0$ or $\tilde{g}_{w}(w, w)<0$. Moreover, $w \in T \tilde{M} \backslash \mathcal{T}$ will be said lightlike (resp. causal, spacelike) if $\tilde{g}_{w}(w, w)=0$ (resp. $\tilde{g}_{w}(w, w) \leq 0$, either $\tilde{g}_{w}(w, w)>0$ or $\left.w=0\right)$. Moreover, assuming that a section $T$ as above can be chosen, we say that a causal vector $w$ is future-pointing (resp. past pointing) if either $w$ is a positive (resp. negative) multiple of $T_{\tilde{\pi}(w)}$ or $\tilde{g}_{w}(w, T)<0$ (resp. $\tilde{g}_{w}(w, T)>0$ ). A continuous piecewise smooth curve $\gamma: I \rightarrow \tilde{M}, I \subseteq \mathbb{R}$, will be called timelike (resp. lightlike, causal, spacelike) if $\dot{\gamma}^{ \pm}(s)$ are timelike (resp. lightlike, causal, spacelike) for all $s \in I$ 
(where, $\dot{\gamma}^{ \pm}(s)$ denotes the right and the left derivatives of $\gamma$ at $s$ ). Moreover, a causal curve $\gamma: I \rightarrow \tilde{M}$ will be said future-pointing, (resp. past pointing) if $\dot{\gamma}^{ \pm}(s)$ are future (resp. past) pointing for all $s \in I$. Finally a smooth embedded hypersurface $\mathcal{H} \subset M$ which is transversal to $\mathcal{T}$ will be called spacelike if for any $v \in T \mathcal{H}$, $\tilde{g}_{v}(v, v)>0$.

Definition 1.7. Let $(\tilde{M}, L)$ be a Finsler spacetime and $\gamma:[a, b) \rightarrow \tilde{M}$ a continuous piecewise smooth future-pointing causal curve. Then $\gamma$ is called future extendible if it has a continuous extension at $b$; it is future inextensible otherwise. Analogously $\gamma:(a, b] \rightarrow \tilde{M}$ is called past extendible if it can be continuously extended at $a$ and it is past inextensible otherwise. Moreover, a future-pointing causal curve $\gamma:(a, b) \rightarrow \tilde{M}$ is inextensible if it is future and past inextensible.

Henceforth, we will always consider continuous piecewise smooth curves, so that we will often omit to specify it when considering a curve.

\section{Standard Static Finsler Spacetimes}

Let us recall that a Lorentzian spacetime $(\tilde{M}, g)$ is said static if it is endowed with an irrotational timelike Killing vector field $K$. This is equivalent to say that the orthogonal distribution to $K$ is locally integrable and then for each $p \in \tilde{M}$ there exists a spacelike hypersurface $S$, orthogonal to $K, p \in S$, and an open interval $I$ such that the pullback of the metric $g$ by a local flow of $K$, defined in $I \times S$, is given by $-\Lambda d t^{2}+g_{0}$, where $t \in I, \partial_{t}$ is the pullback of $K, \Lambda=-g(K, K)$ and $g_{0}$ is the Riemannian metric induced on $S$ by $g$ (see [36, Proposition 12.38]). This local property of static spacetimes justifies the following definition: let $M$ be an $n$-dimensional Riemannian manifold, $\Lambda: M \rightarrow(0,+\infty)$ a smooth, positive function on $M$ and $I \subseteq \mathbb{R}$ an open interval. The warped product $I_{\Lambda} \times M$, i.e. the manifold $I \times M$ endowed with the Lorentzian metric $g=-\Lambda d t^{2}+g_{0}$, where $g_{0}$ is the pullback on $I \times M$ of the Riemannian metric on $M$, is a spacetime called standard static (see [36, Definition 12.36]).

The conformal Riemannian metric on $M, g_{0} / \Lambda$ is called optical metric. It plays a fundamental role in the study of light rays of $I_{\Lambda} \times M$ because its pregeodesics are the projections on $M$ of the light rays in $(I \times M, g)$ (see [3], [21], [37]). Moreover, many of the causal properties of the spacetime $I_{\Lambda} \times M$ are encoded in the geometry of the conformal manifold $\left(M, g_{0} / \Lambda\right)$. For example, global hyperbolicity of $I_{\Lambda} \times M$ and the fact that the the slices $\left\{t_{0}\right\} \times M, t_{0} \in I$, are Cauchy hypersurfaces are both equivalent to the completeness of the optical metric (these properties follows by the conformal invariance of causal properties plus Theorem 3.67 and Theorem 3.69-(1) with $f \equiv 1$ of [8]).

Let us extend the above picture to Finsler spacetimes. First we need the notion of a Killing vector field.

Definition 2.1. Let $(\tilde{M}, L)$ be a Finsler spacetime and $K$ be a vector field on $\tilde{M}$. Let $\psi$ be the flow of $K$. We say that $K$ is a Killing vector field if for each $v \in T \tilde{M} \backslash \mathcal{T}$ and for all $v_{1}, v_{2} \in T_{\pi(v)} \tilde{M}$, we have:

$$
\tilde{g}_{d \psi_{\bar{t}}(v)}\left(d \psi_{\bar{t}}\left(v_{1}\right), d \psi_{\bar{t}}\left(v_{2}\right)\right)=\tilde{g}_{v}\left(v_{1}, v_{2}\right),
$$

for any $\bar{t} \in \mathbb{R}$ such that the stage $\psi_{\bar{t}}$ is well defined in a neighbourhood $U \subset \tilde{M}$ of $\pi(v)$. 
Remark 2.2. As proved in [30, Proposition 5.2], this is equivalent to the fact that $K$ is a Killing vector field for $\tilde{g}$, in the sense that the Lie derivative $\mathcal{L}_{K} \tilde{g}=0$ (see $\left[30\right.$, p.136] for the definition of the Lie derivative over the tensor bundle $\pi^{*}\left(T^{*} \tilde{M}\right) \otimes$ $\pi^{*}\left(T^{*} \tilde{M}\right)$; actually in [30] the base of the tensor bundle is the slit tangent bundle $T \tilde{M} \backslash 0$ but the reader can check the validity of the equivalence when considering $T \tilde{M} \backslash \mathcal{T})$.

Definition 2.3. We say that a Finsler spacetime $(\tilde{M}, L)$ is static if there exists a timelike Killing vector field $K$ such that the distribution of hyperplanes is integrable, where $\partial_{v} L(K)$ denotes the one-form on $\tilde{M}$ given by $\frac{\partial L}{\partial v^{i}}(K) d x^{i}$.

Remark 2.4. Observe that the above definition is well posed since $L$ is at least a $C^{1}$ function on $T \tilde{M}$. In particular, it works also for a smooth global section $T$ of $\mathcal{T}$ (in the case when $\mathcal{T}$ is trivial) or, more generally, for a vector field $K$ such that $K_{p} \in \mathcal{T}_{p}$ for some $p \in \tilde{M}$.

Definition 2.5. We say that a Finsler spacetime is standard static if there exist a smooth non vanishing global section $T$ of $\mathcal{T}$, a Finsler manifold $(M, F)$, a positive function $\Lambda$ on $M$ and a smooth diffeomorphism $f: \mathbb{R} \times M \rightarrow \tilde{M}, f=f(t, x)$, such that $\partial_{t}=f^{*}(T)$ and $L\left(f_{*}(\tau, v)\right)=-\Lambda \tau^{2}+F^{2}(v)$.

Remark 2.6. The definition of a standard static Finsler spacetime (although called there static Finsler spacetime) appeared first in [28, Definition 2]. In [29] the solution of the vacuum (Finslerian) field equations, introduced in the same paper, is standard static in the region where a certain coefficient $B$ is positive provided that a constant $a$ is also positive (see [29, Eqs. (16)-(17)]).

Remark 2.7. Another static future-pointing Killing vector field $K$ will be said standard if the above conditions hold relatively to $K$, i.e there exist a manifold $\left(M^{\prime}, G\right)$ and a diffeomorphism $f^{\prime}: \mathbb{R} \times M^{\prime} \rightarrow \tilde{M}, f^{\prime}=f^{\prime}\left(t^{\prime}, x^{\prime}\right)$, such that $\partial_{t^{\prime}}=\left(f^{\prime}\right)^{*}(K)$ and $L\left(f_{*}^{\prime}\left(\tau^{\prime}, v^{\prime}\right)\right)=-\Lambda^{K} \tau^{\prime 2}+G^{2}\left(v^{\prime}\right)$.

The existence of a standard static vector field is a very rigid condition in comparison to the Lorentzian case $([44,2])$ where some topological assumptions on the base $M$ are needed in order to get uniqueness. Indeed we have the following:

Proposition 2.8. If a static Finsler spacetime admits a standard splitting (i.e the static vector field is standard) then it is unique up to rescaling $t \mapsto t / a, T \mapsto a T$, $a \in(0,+\infty)$, of the coordinate $t$ and of the vector field $T$ and up to Finslerian isometries of $(M, F)$.

Proof. Assume that another static standard Killing vector field $K$ exists and let $f^{\prime}: \mathbb{R} \times M^{\prime} \rightarrow \tilde{M}$ be a smooth diffeomorphism such that $\left(f^{\prime}\right)^{*}(K)=\partial_{t^{\prime}}$ and $L\left(f_{*}^{\prime}\left(\tau^{\prime}, v^{\prime}\right)\right)=-\Lambda^{K} \tau^{\prime 2}+G^{2}\left(v^{\prime}\right)$, so that $G^{2}\left(v^{\prime}\right)=L\left(f_{*}^{\prime}\left(0, v^{\prime}\right)\right)$ for all $v^{\prime} \in T M^{\prime}$. Let $L^{\prime}:=L \circ f_{*}^{\prime}$. Then $L=L^{\prime} \circ\left(f^{\prime}\right)^{*}$, hence $L$ is not twice differentiable along the line bundle $\mathcal{K}$ defined by $K$, because $L^{\prime}$ is not so along the one defined by $\partial_{t^{\prime}}$. This is possible if and only if $\mathcal{K}=\mathcal{T}$, which is equivalent to the fact that $K$ is collinear to $T$ at every point in $\tilde{M}$. Since both $K$ and $T$ are Killing vector fields for $\tilde{g}$ and they belong to the same timelike cone necessarily they are proportional, i.e. there exists a positive constant $a$ such that $K=a T$. This follows as in the semi-Riemannian case, by using the fact that $\mathcal{L}_{T} \tilde{g}=\mathcal{L}_{K} \tilde{g}=0$ (see [30, p. 136, Definition 5.1 and Proposition 5.2]). Thus the diffeomorphism $\left(f^{\prime}\right)^{-1} \circ f$ has first component equal to 
$t \mapsto t / a$ while the second one induces a diffeomorphism $\phi$ between $M$ and $M^{\prime}$ such that $G^{2}\left(\phi_{*}(v)\right)=F^{2}(v)$ for all $v \in T M$.

Remark 2.9. Henceforth, we will identify a standard static Finsler spacetime $(\tilde{M}, L)$ with the product manifold $\mathbb{R} \times M$ endowed with the Finsler function $L(\tau, v)=$ $-\Lambda \tau^{2}+F^{2}(v)$, where $\Lambda$ and $F$ are, respectively, a positive function and a Finsler metric on $M$.

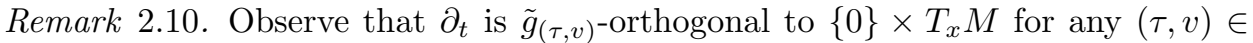
$T \tilde{M} \backslash \mathcal{T}, x=\pi^{M}(v)$, where $\pi^{M}: T M \rightarrow M$ is the canonical projection. These facts justify, by analogy with the Lorentzian case, the name "standard static" given to the class of Finsler spacetime in Definition 2.5. Anyway, as observed in [34, Example 1, Remark 3], differently from the Lorentzian case, a Finsler spacetime can be static without being locally standard static either (in the example of [34], the Killing vector field is not $\tilde{g}_{\tilde{v}}$-orthogonal to $\operatorname{ker}\left(\partial_{v} L\left(K_{\pi(\tilde{v})}\right)\right)$, for all $\left.\tilde{v} \in \operatorname{ker}\left(\partial_{v} L(K)\right)\right)$.

Remark 2.11. Clearly, in Definition 2.5, we could allow more generality by taking a positive definite, homogeneous, generalized metric $g$ on $M$ (recall Remark 1.2) and/or a function $\tilde{\Lambda}: T M \backslash 0 \rightarrow(0,+\infty)$ which is fiberwise positively homogeneous of degree 0 , i.e $\tilde{\Lambda}(\lambda v)=\tilde{\Lambda}(v)$ for each $v \in T M \backslash 0$ and $\lambda>0$. Let us focus on the latter case. Observe, first, that the generalized metric $\tilde{g}$ will not come, in general, from a Finsler function. In such a generalized standard static Finsler spacetime the set of future-pointing causal vectors $J$ at a point $(t, x)$ is given by the non-zero vectors $(\tau, v)$ satisfying $\tau \geq F(v) / \sqrt{\Lambda(v)}$. Being $\Lambda$ positively homogeneous of degree 0 and positive it satisfies $C_{1}(x) \leq \Lambda(v) \leq C_{2}(x)$, for some positive constants $C_{1}(x), C_{2}(x)$, and for all $v \in T_{x} M \backslash\{0\}$, so that $F / \sqrt{\Lambda}$ can be extended by continuity in 0 . Thus, $J$ is connected. Nevertheless, it is, in general, non-convex (see, e.g., Figure 1). This is in contrast to what happens in Finsler spacetimes defined through a Finsler function where the connected components of $J$ are convex (see [34, Theorem 2]) and it should be considered as a serious argument against the definition of a Finsler spacetime through a generalized metric which does not come from a quadratic Finsler function. In fact, in this case, a reverse Cauchy-Schwarz inequality (see Proposition 2.22 below) cannot hold and there exist causal vectors $\left(\tau_{1}, v\right),\left(\tau_{2}, w\right)$ which are in the same connected component of the set of causal vectors and such that $\tilde{g}_{\left(\tau_{1}, v_{1}\right)}\left(\left(\tau_{1}, v_{1}\right),\left(\tau_{2}, w\right)\right)>0$ (see Figure 1$)$.

Let us determine the geodesic equations in a standard static Finsler spacetime. Given a manifold $N$ and two points $p, q \in N$, let $\Omega_{p q}(N)$ be the set of the continuous piecewise smooth curve $\gamma$ on $N$ parametrized on a given interval $[a, b] \subset \mathbb{R}$ and connecting $p$ to $q$ (i.e. $\gamma(a)=p, \gamma(b)=q$ ). If $\gamma \in \Omega_{p q}(N)$, we call a (proper) variation of $\gamma$ a continuous two-parameter map $\psi:(\varepsilon, \varepsilon) \times[a, b] \rightarrow N$ such that $\psi(0, s)=\gamma(s)$, for all $s \in[a, b], \psi(w, \cdot) \in \Omega_{p q}(N)$ and there exists a subdivision $a=s_{0}<s_{1}<\ldots, s_{k}=b$ of the interval $[a, b]$ for which $\left.\psi\right|_{(-\varepsilon, \varepsilon) \times\left[s_{j-1}, s_{j}\right]}$ is smooth for all $j \in\{1, \ldots, k\}$. Clearly, we can define classes of proper variations of $\gamma$ as those sharing the same variational vector field $Z$. This is, by definition, a continuous piecewise smooth vector field along $\gamma$ such that $Z(a)=0=Z(b)$ and $Z(s)=$ $\frac{\partial \psi}{\partial r}(0, s)$. By considering any auxiliary Riemannian metric $h$ on $N$, we see that each variational vector field $Z$ along $\gamma$ individuates a variation (and then also a class of them) by setting $\psi(w, s):=\exp _{\gamma(s)}(w Z(s))$, for $|w|<\varepsilon$ small enough. 


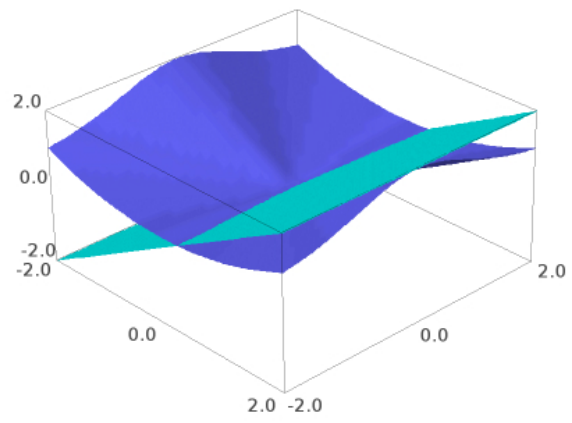

Figure 1 . The set of the f. p. lightlike vectors (in blue) in $\mathbb{R} \times \mathbb{R}^{2}$ with the (flat) static metric $\tilde{g}=-e^{\frac{4 v_{2}^{2}}{v_{1}^{2}+v_{2}^{2}}} d t^{2}+d x^{2}+d y^{2}$. In cyan, it is represented the plane of vectors $\left(\tau, v_{1}, v_{2}\right)$ which are $\tilde{g}_{(1,1,0)^{-}}$ orthogonal to the lightlike vector $(1,1,0)$.

Let us consider the energy functional

$$
E: \Omega_{p q}(\tilde{M}) \rightarrow \mathbb{R}, \quad E(\gamma)=\frac{1}{2} \int_{a}^{b}\left(-\Lambda(\sigma) \dot{\zeta}^{2}+F^{2}(\dot{\sigma})\right) d s .
$$

As $\tilde{M}$ splits as $\mathbb{R} \times M$, the path space $\Omega_{p q}(\tilde{M})$ is identifiable with the product $\Omega_{t_{p} t_{q}}(\mathbb{R}) \times \Omega_{x_{p} x_{q}}(M)$, where $\left(t_{p}, x_{p}\right)=p$ and $\left(t_{q}, x_{q}\right)=q$ and any curve $\gamma \in \Omega_{p q}(\tilde{M})$ has two components $\gamma(s)=(\theta(s), \sigma(s))$.

Definition 2.12. A continuous piecewise smooth curve $\gamma:[a, b] \rightarrow \tilde{M}$ is a (affinely parametrized) geodesic of $(\tilde{M}, L)$ if it is a critical point of the energy functional, i.e. if $\frac{d}{d r}\left(\left.E(\psi(r, \cdot))\right|_{r=0}=0\right.$, for all proper variations $\psi$ of $\gamma$.

Theorem 2.13. A curve $\gamma:[a, b] \rightarrow \tilde{M}, \gamma(s)=(\theta(s), \sigma(s))$, is a geodesic of $(\tilde{M}, L)$ if and only if the following equations are satisfied in local natural coordinates $\left(t, x^{1}, \ldots, x_{n}, \tau, v^{1}, \ldots, v^{n}\right)$ on $T \tilde{M}$ :

$$
\begin{aligned}
- & \frac{\partial \Lambda}{\partial x^{i}}(\sigma) \dot{\theta}^{2}+\frac{\partial F^{2}}{\partial x^{i}}(\dot{\sigma})-\frac{d}{d s}\left(\frac{\partial F^{2}}{\partial v^{i}}(\dot{\sigma})\right)=0, \quad i=1, \ldots, n \\
& \Lambda(\sigma) \dot{\theta}=\text { const. }
\end{aligned}
$$

$\sigma$ and $\theta$ are, respectively, $C^{1}$ and $C^{2}$ on $[a, b]$ and there exists a constant $C \in \mathbb{R}$ such that $L(\dot{\gamma}(s))=C$, for all $s \in[a, b]$.

Moreover, if $\gamma$ is a non-constant geodesic then: (a) if it is spacelike or lightlike (i.e. $C \geq 0$ ) then $\dot{\sigma}$ never vanishes and $\gamma$ is smooth; (b) if $\sigma$ is constant equal to $x_{0} \in M$ on the whole interval $[a, b]$ then $C<0, d \Lambda\left(x_{0}\right)=0$ and $\dot{\theta}$ is constant too; vice versa, if $d \Lambda\left(x_{0}\right)=0$ then, for each $\theta_{0} \in \mathbb{R}$ and $m \neq 0$, the curve $s \in[a, b] \mapsto$ $\left(\theta_{0}+m(s-a), x_{0}\right) \in \tilde{M}$ is a timelike geodesic. 
Remark 2.14. In particular the function $s \in[a, b] \mapsto \frac{\partial F^{2}}{\partial v^{i}}(\dot{\sigma}(s))$ is $C^{1}$ on $[a, b]$, in fact it is differentiable also at the instants $s$ where $\dot{\sigma}(s)=0$.

Proof. By considering variational vector fields $Z$ which are, respectively, of the type $(Y, 0)$ and $(0, W)$ and have compact support in any open maximal interval $I$ where $\sigma$ is smooth and $\dot{\sigma}(s) \neq 0$, we deduce by standard arguments that in local coordinates $\left(t, x^{1}, \ldots, x^{n}, \tau, v^{1}, \ldots, v^{n}\right)$ of $T \tilde{M}$, any critical point $\gamma=(\theta, \sigma)$ of $E$ satisfies, in such interval $I$, equations (2) and (3). Actually, it can be easily seen that (3) holds on all $[a, b]$ and then, as $\sigma$ is continuous, $\theta$ is $C^{1}$ on $[a, b]$. If $\bar{s} \in[a, b]$ is an isolated zero of $\dot{\sigma}(s)$, and $\sigma$ is smooth in a neighbourhood $J$ of $\bar{s}$ then, as the functions $-\frac{1}{2} \frac{\partial \Lambda}{\partial x^{i}}(\sigma) \dot{\theta}^{2}+\frac{\partial F^{2}}{\partial x^{i}}(\dot{\sigma})$ and $\frac{\partial F^{2}}{\partial v^{i}}(\dot{\sigma}(s))$ are continuous in $J$, we deduce, from the fact that (2) is satisfied in a left and in a right neighbourhood of $\bar{s}$, that $s \mapsto \frac{\partial F^{2}}{\partial v^{i}}(\dot{\sigma}(s))$ is differentiable with continuous derivative at $\bar{s}$ and (2) is satisfied also at $\bar{s}$. By this information at isolated zeroes of $\dot{\sigma}$, we see that the same argument applies at a zero where $\sigma$ is smooth and which is an accumulation point of isolated zeroes.

On the other hand, at the instants $s_{j}, j \in\{0, \ldots, k\}$, where $\dot{\sigma}$ has a break, by taking any vector $w_{j} \in T_{\sigma\left(s_{j}\right)} M, j \in\{1, \ldots, k-1\}$, and a variational vector field $\left(0, W_{j}\right)$, such that $W_{j}\left(s_{j}\right)=w_{j}$ and $W \equiv 0$ outside a small neighbourhood of $s_{j}$, we get, using that $(2)$ is satisfied both in $\left[s_{j-1}, s_{j}\right]$ and $\left[s_{j}, s_{j+1}\right]$,

$$
\frac{\partial F^{2}}{\partial v^{i}}\left(\dot{\sigma}^{-}\left(s_{j}\right)\right) w_{j}^{i}=\frac{\partial F^{2}}{\partial v^{i}}\left(\dot{\sigma}^{+}\left(s_{j}\right)\right) w_{j}^{i},
$$

hence $\frac{\partial F^{2}}{\partial v}\left(\dot{\sigma}^{-}\left(s_{j}\right)\right)=\frac{\partial F^{2}}{\partial v}\left(\dot{\sigma}^{+}\left(s_{j}\right)\right)$. Being the map $v \in T_{x} M \mapsto \frac{\partial F^{2}}{\partial v}(v) \in T_{x}^{*} M$ a homeomorphism (see e.g. [13, p.373]) we deduce that $\dot{\sigma}^{-}\left(s_{j}\right)=\dot{\sigma}^{+}\left(s_{j}\right)$. Thus, $\sigma$ is a $C^{1}$ curve on $[a, b]$ and, from (3), $\theta$ is a $C^{2}$ function. Then, from $s$-independence of the Lagrangian $L$ and the fact that it is positively homogeneous of degree 2 , we know that $L(\dot{\gamma}(s))=\frac{\partial L}{\partial v}(\dot{\gamma}(s))[\dot{\gamma}(s)]-L(\dot{\gamma}(s))=$ const. $:=C_{j} \in \mathbb{R}$ in each interval $\left[s_{j}, s_{j+1}\right]$ where $\gamma$ is smooth. As $\gamma$ is a $C^{1}$ curve on $[a, b]$, the constants $C_{j}$ must agree, i.e. $L(\dot{\gamma}(s))=$ const. $:=C \in \mathbb{R}$ on $[a, b]$.

The converse clearly follows by observing that each $C^{1}$ curve $\gamma$ which solves (2) and (3) must necessarily be a critical point of the energy functional.

Last part of the theorem follows by observing that, being $\Lambda>0$, if $C>0$ then $\dot{\sigma} \neq 0$ everywhere in $[a, b]$ and if $\gamma$ is non-constant and $C=0$ then at each instant $\bar{s} \in[a, b]$ where $\dot{\sigma}(\bar{s})=0$, necessarily, also $\dot{\theta}(\bar{s})=0$ and, from (3), $\dot{\theta} \equiv 0$ and also $F^{2}(\dot{\sigma}) \equiv 0$, i.e. $\dot{\sigma} \equiv 0$, a contradiction since $\gamma$ was not constant. As the vertical Hessian of $F^{2}$ is invertible at each non-zero vector, from (2) written in normal form, we deduce that if $C \geq 0$ then $\sigma$ is smooth (i.e. it is at least twice differentiable also at the instants $s_{j}, j \in\{1, \ldots, k\}$. Finally, if $\sigma$ is constant and equal to $x_{0} \in M$ then necessarily $C<0$ and, from (3), $\dot{\theta}$ is a non-zero constant while, from (2), $\frac{\partial \Lambda}{\partial x^{i}}\left(x_{0}\right)=0$. Analogously, one can check that last statement holds true.

Definition 2.15. A pregeodesic of $(\tilde{M}, L)$ is any $C^{1}$ curve $\gamma:[c, d] \rightarrow \tilde{M}$ admitting a reparametrization $\varphi:[a, b] \rightarrow[c, d]$ which is $C^{1}$, regular and orientation preserving (i.e. $\dot{\varphi}>0$ ) such that $\gamma \circ \varphi$ is a geodesic.

As lightlike vectors $w=(v, \tau)$ of $(\mathbb{R} \times M, \tilde{g})$ are defined by the equation $g_{v}(v, v)-$ $\Lambda \tau^{2}=0$, we give, in analogy to the Lorentzian case, the following definition:

Definition 2.16. The optical "metric" of a standard static Finsler spacetime is the positive definite homogeneous section $v \in T M \backslash 0 \mapsto \frac{1}{\Lambda} g_{v}$. 
Remark 2.17. Notice that the optical metric is the fundamental tensor of the Finsler metric $\tilde{F}=F / \sqrt{\Lambda}$ on $M$.

Definition 2.16 is justified by the following result:

Proposition 2.18. Let $(\tilde{M}, L)$ be a standard static Finsler spacetime. A curve $\gamma:[a, b] \rightarrow \tilde{M}, \gamma(s)=(\theta(s), \sigma(s))$ is a future-pointing lightlike geodesic if and only if $\sigma$ is a (non-constant) pregeodesic of the Finsler metric $F / \sqrt{\Lambda}$ on $M$ parametrized with $\sqrt{\Lambda(\sigma)} F(\dot{\sigma})=$ const. and $\theta(s)=\theta(a)+\int_{a}^{s} \frac{F(\dot{\sigma})}{\sqrt{\Lambda(\sigma)}} d \tau$.

Proof. Let us assume that $\gamma$ is a future-pointing lightlike geodesic of $(\tilde{M}, L)$. By (3) and the fact that $\gamma$ is lightlike we get that $\sqrt{\Lambda(\sigma)} F(\dot{\sigma})$ must be constant on $[a, b]$. Hence, Eq. (2) is equivalent to

$$
\begin{aligned}
2 \sqrt{\Lambda(\sigma)} F(\dot{\sigma})\left(-\frac{1}{2} \frac{\partial \Lambda}{\partial x^{i}}(\sigma) \frac{F(\dot{\sigma})}{(\Lambda(\sigma))^{3 / 2}}+\frac{1}{\sqrt{\Lambda(\sigma)}} \frac{\partial F}{\partial x^{i}}(\dot{\sigma})\right. & \\
& \left.-\frac{d}{d s}\left(\frac{1}{\sqrt{\Lambda(\sigma)}} \frac{\partial F}{\partial v^{i}}(\dot{\sigma})\right)\right)=0 .
\end{aligned}
$$

As $\sqrt{\Lambda(\sigma)} F(\dot{\sigma})$ is positive, the above equation is satisfied if and only if

$$
-\frac{1}{2} \frac{\partial \Lambda}{\partial x^{i}}(\sigma) \frac{F(\dot{\sigma})}{(\Lambda(\sigma))^{3 / 2}}+\frac{1}{\sqrt{\Lambda(\sigma)}} \frac{\partial F}{\partial x^{i}}(\dot{\sigma})-\frac{d}{d s}\left(\frac{1}{\sqrt{\Lambda(\sigma)}} \frac{\partial F}{\partial v^{i}}(\dot{\sigma})\right)=0 .
$$

This is the Euler-Lagrange equation (in natural local coordinates of $T M$ ) of the length functional associated to $F / \sqrt{\Lambda}$, hence $\sigma$ is a pregeodesic of the Finsler manifold $(M, F / \sqrt{\Lambda})$. The converse immediately follows by using invariance under $C^{1}$, regular, orientation preserving reparametrizations of the solutions of (4).

Remark 2.19. Notice that the length functional of the Finsler metric $F / \sqrt{\Lambda}$ on the path space $\Omega_{x_{p} x_{q}}(M)$ coincides, up to a constant, with the arrival time functional $T_{p l_{x_{q}}}$ of the standard static Finsler spacetime $(\tilde{M}, L)$; this is the functional defined on the set of the future-pointing lightlike curves $\gamma$ connecting $p$ with the line $l_{x_{q}}=$ $s \mapsto\left(s, x_{q}\right)$ and defined as $T_{p l_{x_{q}}}(\gamma)=t(\gamma(b))$. Hence, Proposition 2.18 can be interpreted as a Fermat's principle for light rays in the Finsler spacetime $(\tilde{M}, L)$, namely the critical point of $T_{p l_{x_{q}}}$ are all and only the lightlike pregeodesic of $(\tilde{M}, L)$. For a general version of the Fermat's principle in a Finsler spacetime defined through a quadratic Finsler function which is smooth on $T \tilde{M} \backslash 0$ see [38, Section 4].

Remark 2.20. Let $\tilde{L}: T \tilde{M} \rightarrow \mathbb{R}$ be the function given by $\tilde{L}(\tau, v)=\frac{1}{\Lambda} F^{2}(v)-\tau^{2}$. We call the pair $(\tilde{M}, \tilde{L})$ the ultrastatic Finsler spacetime associated to $(\tilde{M}, L)$ and we denote by $G$ the square of $\tilde{F}: G=\frac{1}{\Lambda} F^{2}$ and by $\tilde{d}$ the distance associated to $\tilde{F}$. From Proposition 2.18 we immediately deduce that the Finsler spacetime $(\tilde{M}, L)$ and the ultrastatic one $(\tilde{M}, \tilde{L})$ share the same lightlike pregeodesics. In other words, invariance of lightlike geodesics under conformal changes of the metric (which is a fundamental property of Lorentzian spacetime) also holds in the class of standard static Finsler spacetime under conformal factors depending only on $x \in M$.

It has been known at least since [7] that the causal structure of a tangent space in a Finsler spacetime can be weird. In fact, [7] contains some two-dimensional 
examples of Finsler spacetime where the causal cone at a point $x$ (i.e. the the set of the vectors $v \in T_{x} \tilde{M} \backslash\{0\}$ which are causal) has more than two connected component. Recently, in [34], it has been proved that such pathologies are confined in dimension two if the Finsler spacetime is time oriented and the Finsler spacetime metric is reversible. For a standard static spacetime $(\tilde{M}, L)$ things are simpler as the following proposition shows:

Lemma 2.21. Let $(\tilde{M}, L)$ be a standard static Finsler spacetime. Then the set of future-pointing causal vectors at a point $(t, x) \in \tilde{M}$ has only one connected convex component. Moreover, for each $c>0$, the set $J(c)$ of the future-pointing timelike vectors in $T_{(t, x)} \tilde{M}$ such that $\left.L(\tilde{v}) \leq-c\right\}$ is also connected and strictly convex.

Proof. Observe that, by definition, future-pointing causal vectors $\tilde{v}=(\tau, v) \in$ $T_{(t, x)} \tilde{M}$ are all and only the non-zero vectors satisfying $\tau \geq \tilde{F}(v)$ (recall that, for all $\tilde{v} \in T \tilde{M} \backslash \mathcal{T}$, as $\tilde{g}_{\tilde{v}}\left(\tilde{v}, \frac{\partial}{\partial t}\right) \leq 0, \tau$ has non-negative sign). Being $\tilde{F}$ continuous on $T_{x} M$ and fiberwise convex (see, e.g [46, Lemma 1.2.2]) its epigraph in $T_{x} M$ is connected and convex. For the last part of the proposition, observe that $\tilde{v} \in J(c)$ if and only if $\tau \geq \sqrt{G(v)+\alpha}$ where $\alpha=c / \Lambda(x)$. A simple computation shows that for each $v \in T_{x} M \backslash\{0\}$, the fiberwise Hessian of $\sqrt{G+\alpha}$ at $v$ is given by

$$
\frac{\frac{\partial \tilde{F}}{\partial v^{i}}(v) \frac{\partial \tilde{F}}{\partial v^{j}}(v)}{\sqrt{G(v)+\alpha}}\left(1-\frac{G(v)}{G(v)+\alpha}\right)+\frac{\tilde{F}(v)}{\sqrt{G(v)+\alpha}} \frac{\partial^{2} \tilde{F}}{\partial v^{i} \partial v^{j}}(v) .
$$

Since $\frac{\partial^{2} \tilde{F}}{\partial v^{i} \partial v^{j}}(v) w^{i} w^{j} \geq 0$ and it is equal to 0 if and only if $w=\lambda v$, for some $\lambda \in \mathbb{R}$ and, moreover, $\frac{\partial \tilde{F}}{\partial v^{i}}(v) v^{i}=F(v)>0$, we get that the Hessian of $\sqrt{G+\alpha}$ is positive definite for any $v \in T_{x} M \backslash 0$. At $v=0$, observe that the differential of $\sqrt{G+\alpha}$ is 0 and $\sqrt{G(v)+\alpha}>\sqrt{\alpha}=\sqrt{G(0)+\alpha}$. Thus, being $\sqrt{G+\alpha}$ a $C^{1}$ function on $T_{x} M$ we conclude that it is strictly convex and its epigraph is also (connected) and strictly convex.

By Lemma 2.21 we get the following reverse Cauchy-Schwarz inequality:

Proposition 2.22. Let $(\tilde{M}, L)$ be a standard static spacetime and $\tilde{v}, \tilde{w} \in T_{(t, x)} \tilde{M}$, future-pointing causal vectors. Then

$$
-\frac{1}{2} \frac{\partial L}{\partial \tilde{v}^{i}}(\tilde{v}) \tilde{w}^{i} \geq \sqrt{-L(\tilde{v})} \sqrt{-L(\tilde{w})},
$$

with equality if and only if $\tilde{v}$ and $\tilde{w}$ are proportional.

Proof. Recalling that $L$ is $C^{1}$ in $T \tilde{M}$ and smooth outside $\mathcal{T}$, the same proof of [34, Theorem 3] gives

$$
-\tilde{g}_{\tilde{v}}(\tilde{v}, \tilde{w}) \geq \sqrt{-L(\tilde{v})} \sqrt{-L(\tilde{w})},
$$

for any $\tilde{v} \in T_{(t, x)} \tilde{M} \backslash \mathcal{T}_{(t, x)}$ and $\tilde{w} \in T_{(t, x)} \tilde{M}$, with equality if only if $\tilde{v}$ and $\tilde{w}$ are proportional. Then (5) extends to any $\tilde{v} \in T_{(t, x)} \tilde{M}$ by continuity, recalling that, by homogeneity, $-\frac{1}{2} \frac{\partial L}{\partial \tilde{v}^{i}}(\tilde{v}) \tilde{w}^{i}=-\tilde{g}_{\tilde{v}}(\tilde{v}, \tilde{w})$. Moreover if $\tilde{v} \in \mathcal{T}$, so $\tilde{v}=\left(\tau_{1}, 0\right)$ for some $\tau_{1}>0$ then (5) can hold with equality if and only if $\tilde{w} \in \mathcal{T}$. In fact if $\tilde{w}=\left(\tau_{2}, w\right)$, with $w \neq 0$, then the right-hand side of (5) is equal to $\tau_{1} \sqrt{\Lambda(x)} \sqrt{\Lambda(x) \tau_{2}^{2}-F^{2}(w)}$ which is strictly less than the left-hand side equal to $\Lambda(x) \tau_{1} \tau_{2}$.

Remark 2.23. In particular, the above proposition implies that if $\tilde{v}, \tilde{w} \in T_{(t, x)} \tilde{M} \backslash$ $\mathcal{T}_{(t, x)}$, are future-pointing causal vectors then $\tilde{g}_{\tilde{w}}(\tilde{w}, \tilde{v}) \leq 0$ and $\tilde{g}_{\tilde{v}}(\tilde{v}, \tilde{w}) \leq 0$; moreover $\tilde{g}_{\tilde{v}}(\tilde{v}, \tilde{w})=0$ if and only $\tilde{v}$ and $\tilde{w}$ are proportional and lightlike. 


\section{Causality}

In a Lorentzian spacetime $(M, g)$, two events $p, q \in M$ are said chronologically (resp. causally) related and denoted with $p \ll q$ (resp. $p \leq q$ ), if there exists a future-pointing timelike (resp. causal) curve $\gamma$ from $p$ to $q ; p$ is said strictly causally related to $q$, denoted with $p<q$, if $p \leq q$ and $p \neq q$. The chronological (resp. causal) future of $p \in M$ is defined as $I^{+}(p):=\{q \in M: p \ll q\}\left(J^{+}(p)=\right.$ $\{q \in M: p \leq q\})$. Analogous notions appear reversing the binary relation $\ll$, namely we have the chronological (resp. causal) past $I^{-}(p)=\{q \in M: q \ll p\}$ $\left(J^{-}(p)=\{q \in M: q \leq p\}\right)$. For further details see [8], [36].

The above definitions can be trivially extended to a Finsler spacetime $(\tilde{M}, \tilde{g})$ but notice that in the Lorentzian setting the chronological and the causal past of an event $p$ can be equivalently defined by considering past-pointing timelike and causal curves starting at $p$. Clearly, in a Finsler spacetime, this is true only when the generalized metric is absolutely homogeneous, i.e. $\tilde{g}_{v}=\tilde{g}_{-v}$ for any $v \in T \tilde{M} \backslash \mathcal{T}$. In the general case, given a future-pointing causal vector $v$ or a future-pointing causal curve, $-v$ and the curve parametrized with the opposite orientation can be spacelike. So the chronological (resp. causal) past of an event $p$ will be defined by considering only future-pointing timelike (resp. causal) curves arriving at $p$.

One immediate consequence of the definitions of the chronological and the causal future of a point is that, for all $p \in \tilde{M}$ :

$$
I^{+}(p) \subset J^{+}(p), \quad I^{-}(p) \subset J^{-}(p) .
$$

Remark 3.1. After [13] and mostly [14, 12], it is clear that Finsler geometry plays a prominent role in the description of the causal structure of a class of stationary spacetime which generalizes the standard static one in the following sense: under the same notation as in Section 2, let us also consider a one-form on $M$; then define a Lorentzian metric $g$ on the product $\mathbb{R} \times M$ as $g=g_{0}+\omega \otimes d t+d t \otimes \omega-\Lambda d t^{2}$, where $\omega$ is the pullback on $\mathbb{R} \times M$ of the one form on $M$. A Finsler metric $R$ of Randers type emerges then as the optical metric of $(\mathbb{R} \times M, g), R=\frac{1}{\Lambda}\left(\omega+\sqrt{\Lambda g_{0}+\omega \otimes \omega}\right)$. Analogously, the metric structure of the Finsler manifold $(M, F / \sqrt{\Lambda})$ associated to a static Finsler spacetime $(\tilde{M}, L)$ can be related to its causal structure. As a first example, the following proposition analogous to [14, Prop. 4.2] holds:

Proposition 3.2. Let $(\tilde{M}, L)$ be a standard static Finsler spacetime. For all $p_{0}=$ $\left(t_{0}, x_{0}\right) \in \tilde{M}$ we have:

$$
\begin{aligned}
& I^{+}\left(p_{0}\right)=\bigcup_{r>0}\left(\left\{t_{0}+r\right\} \times B^{+}\left(x_{0}, r\right)\right), \\
& I^{-}\left(p_{0}\right)=\bigcup_{r>0}\left(\left\{t_{0}-r\right\} \times B^{-}\left(x_{0}, r\right)\right),
\end{aligned}
$$

where $B^{+}\left(x_{0}, r\right)$ and $B^{-}\left(x_{0}, r\right)$ denote, respectively, the forward and the backward open ball (see $[5, \S 6.2 \mathrm{~B}]$ ) of centre $x_{0}$ and radius $r$ of the Finsler metric $\tilde{F}=F / \sqrt{\Lambda}$. Moreover, $I^{ \pm}\left(p_{0}\right)$ are open subsets of $\tilde{M}$.

Proof. Let us reasoning only for $I^{+}$as the statements for $I^{-}$can be proved analogously. Let $(t, x) \in I^{+}\left(t_{0}, x_{0}\right)$ and $\gamma(s)=(\theta(s), \sigma(s))$ be a timelike curve joining $\left(t_{0}, x_{0}\right)$ to $(t, x)$, so we have that $\theta$ is an increasing function and $\tilde{F}(\dot{\sigma})<\dot{\theta}$. Integrating this inequality, we get $\tilde{d}\left(x_{0}, x\right)<t-t_{0}$. Hence, $x \in B^{+}\left(x_{0}, t-t_{0}\right)$ and we conclude $(t, x) \in\left\{t_{0}+r\right\} \times B^{+}\left(x_{0}, r\right), r=t-t_{0}$. Conversely, let $x \in B^{+}\left(x_{0}, r\right)$, for 
some $r>0$, and $\sigma:[0, a] \rightarrow M$ be a unit $(F / \sqrt{\Lambda})$-speed curve joining $x_{0}$ to $x$, such that $a<r$. The curve $\gamma(s)=\left(t_{0}+\frac{r}{a} s, \sigma(s)\right)$ is then future-pointing, timelike and connects the points $\left(t_{0}, x_{0}\right)$ and $\left(t_{0}+r, x\right)$.

For the last statement observe that if $p=(t, x) \in I^{+}\left(p_{0}\right)$ then $x \in B^{+}\left(x_{0}, t-t_{0}\right)$. Let $\left.\varepsilon=\left(t-t_{0}-\tilde{d}\left(x_{0}, x\right)\right) / 2\right)$. Then one can easily check, using (7), that the open set $(t-\varepsilon,+\infty) \times B^{+}(x, \varepsilon)$ is contained in $I^{+}\left(p_{0}\right)$.

As claimed by Barrett O'Neill [36, p. 293], a fundamental problem in a Lorentzian manifold is to determine which pairs of points can be joined by a timelike curve. Our aim, next, is to extend to standard static Finsler spacetimes [36, Prop. 10.46] stating that there are timelike curves from $p$ to $q$ arbitrarily near to every causal curve $\gamma$ which is not a lightlike pregeodesic. This properties has been already proved in [1, Prop. 7.6], anyway we give here an elementary proof that exploits the splitting $\mathbb{R} \times M$.

Let $\gamma:[a, b] \rightarrow \tilde{M}, \gamma(s)=(\theta(s), \sigma(s))$ be a curve and $\psi:(-\varepsilon, \varepsilon) \times[a, b] \rightarrow \tilde{M}$, $\psi(w, s)=(\zeta(w, s), \eta(w, s))$, be a variation of $\gamma$ with variational vector field $W=$ $(Y, Z)$. Let us denote by $\psi_{w}, w \in(-\varepsilon, \varepsilon)$, the (longitudinal) curve in the variation given by $\psi_{w}:[a, b] \rightarrow \tilde{M}, \psi_{w}(s)=\psi(w, s)$ and by $\dot{\psi}_{w}$ its velocity, $\dot{\psi}_{w}=\frac{\partial}{\partial s} \psi(w, s)$.

Lemma 3.3. Let $\gamma$ be a continuous piecewise smooth causal curve and $\psi$ a variation of $\gamma$ such that $\left.\frac{\partial}{\partial w} L\left(\dot{\psi}_{w}\right)\right|_{w=0}<0$ then, for sufficiently small $w>0$, the associated longitudinal curve $\psi_{w}$ is timelike.

Proof. As

$$
\left.\frac{\partial}{\partial w} \tilde{g}\left(\dot{\psi}_{w}, \dot{\psi}_{w}\right)\right|_{w=0}=\left.\frac{\partial}{\partial w} L\left(\dot{\psi}_{w}\right)\right|_{w=0}<0 .
$$

and $\tilde{g}(\dot{\gamma}, \dot{\gamma}) \leq 0$ the thesis immediately follows.

In any chart $\left((\mathbb{R} \times U) \times\left(\mathbb{R} \times \mathbb{R}^{n}\right),\left(t, x^{1}, \ldots, x^{n}, \tau, v^{1}, \ldots, v^{n}\right)\right)$ of $T \tilde{M}$, such that $\gamma([a, b]) \cap(\mathbb{R} \times U) \neq \emptyset$, we have

$$
\begin{aligned}
\frac{\partial}{\partial w} L\left(\dot{\psi}_{w}\right) & =\frac{\partial}{\partial w}\left(-\Lambda\left(\eta_{w}\right) \dot{\zeta}_{w}^{2}+F^{2}\left(\dot{\eta}_{w}\right)\right) \\
& =\left(-\frac{\partial \Lambda}{\partial x^{i}}\left(\eta_{w}\right) \dot{\zeta}_{w}^{2}+\frac{\partial F^{2}}{\partial x^{i}}\left(\dot{\eta}_{w}\right)\right) \frac{\partial \eta_{w}^{i}}{\partial w}-2 \Lambda\left(\eta_{w}\right) \dot{\zeta}_{w} \frac{\partial \dot{\zeta}_{w}}{\partial w}+\frac{\partial F^{2}}{\partial v^{i}}\left(\dot{\eta}_{w}\right) \frac{\partial \dot{\eta}_{w}^{i}}{\partial w}
\end{aligned}
$$

up to the finite number of instants $s_{j}$ where, eventually, $\dot{\gamma}$ has breaks (clearly, the above equation is satisfied, separately, in some intervals of the type $\left(s_{j}-\varepsilon_{1 j}, s_{j}\right]$, $\left[s_{j}, s_{j}+\varepsilon_{2 j}\right), \varepsilon_{1 j}, \varepsilon_{2 j}>0$ such that $\left.\gamma\left(\left(s_{j}-\varepsilon_{1 j}, s_{j}+\varepsilon_{2 j}\right)\right) \subset \mathbb{R} \times U\right)$.

For $w=0$, the above equation yields

$$
\left.\frac{\partial}{\partial w} L\left(\dot{\psi}_{w}\right)\right|_{w=0}=\left(-\frac{\partial \Lambda}{\partial x^{i}}(\sigma) \dot{\theta}^{2}+\frac{\partial F^{2}}{\partial x^{i}}(\dot{\sigma})\right) Z^{i}-2 \Lambda(\sigma) \dot{\theta} \dot{Y}+\frac{\partial F^{2}}{\partial v^{i}}(\dot{\sigma}) \dot{Z}^{i} .
$$

Now we consider the following equation in each coordinate system as above:

$$
\frac{\partial F^{2}}{\partial v^{i}}(\dot{\sigma}) \dot{Z}^{i}+\left(\frac{\partial F^{2}}{\partial x^{i}}(\dot{\sigma})-\frac{\partial \Lambda}{\partial x^{i}}(\sigma) \dot{\theta}^{2}\right) Z^{i}-2 \Lambda(\sigma) \dot{\theta} \dot{Y}=-\alpha
$$

where $\alpha$ is a positive constant.

Proposition 3.4. Let $\rho:[a, b] \rightarrow \tilde{M}, \rho(s)=\left(\rho_{1}(s), \rho_{2}(s)\right)$, be a future-pointing causal curve of $(\tilde{M}, L)$ that is not a lightlike pregeodesic, then there exists a proper variation of $\rho$ by timelike curves. 
Proof. If $\rho$ is a timelike curve then the thesis follows by a simple continuity argument. Then assume that $\rho$ is not timelike. Being $\rho$ causal and future-pointing, $\Lambda\left(\rho_{2}\right) \dot{\rho}_{1}>0$ on $[a, b]$, thus we can reparametrize $\rho$ on the same interval $[a, b]$ to obtain a curve $\gamma=\gamma(s)=(\theta(s), \sigma(s))$ such that $\Lambda(\sigma) \dot{\theta}=C$ for some positive constant $C$. Let us consider a covering $\left\{\mathbb{R} \times U_{i}\right\}_{i \in\{1, \ldots, k\}}$ of $\gamma$ by $k$ charts of $\tilde{M}$ and a subdivision $a=s_{0}<s_{1}<\ldots<s_{k}$ of the interval $[a, b]$ such that $\gamma\left(\left[s_{j-1}, s_{j}\right]\right) \subset \mathbb{R} \times U_{j}$ for all $j \in\{1, \ldots, k\}$. As $\rho$ was not a lightlike pregeodesic, necessarily it must exist a piecewise smooth vector field $Z$ along $\sigma$, with $Z(a)=Z(b)=0$ such that

$$
\sum_{j=1}^{k} \int_{s_{j-1}}^{s_{j}}\left(\frac{\partial F^{2}}{\partial v^{i}}(\dot{\sigma}) \dot{Z}^{i}+\left(\frac{\partial F^{2}}{\partial x^{i}}(\dot{\sigma})-\frac{\partial \Lambda}{\partial x^{i}}(\sigma) \dot{\theta}^{2}\right) Z^{i}\right) d s \neq 0,
$$

otherwise, from (2), $\gamma$ would be a geodesic of $(\tilde{M}, L)$ and then (being causal and not timelike) necessarily a lightlike one. Clearly, up to consider the opposite vector $-Z$, we can assume that the above summation is negative. Let us define on the coordinate system associated to $\mathbb{R} \times U_{j}$

$$
h_{j}=\frac{\partial F^{2}}{\partial v^{i}}(\dot{\sigma}) \dot{Z}^{i}+\left(\frac{\partial F^{2}}{\partial x^{i}}(\dot{\sigma})-\frac{\partial \Lambda}{\partial x^{i}}(\sigma) \dot{\theta}^{2}\right) Z^{i}, \quad j \in\{1, \ldots, k\}
$$

then

$$
Y(s)=\frac{1}{2 C}\left(\sum_{j=1}^{m(s)} \int_{s_{j-1}}^{s_{j}} h_{j}(\mu) d \mu+\int_{s_{m(s)}}^{s} h_{m(s)+1} d \mu+\alpha(s-a)\right),
$$

where $m(s) \in\{0, \ldots, k-1\}$, such that $s \in\left(s_{m(s)}, s_{m(s)+1}\right]$ (with the convention that if $m(s)=0$ then the first term in the right-hand side is equal to 0 ), solves (9) and for $\alpha=-\frac{1}{b-a} \sum_{j=1}^{k} \int_{s_{j-1}}^{s_{j}} h_{j}(\mu) d \mu$ we get that $\alpha>0$ and $Y(b)=0$. From (8), this implies that

$$
\left.\frac{\partial}{\partial w} L\left(\dot{\psi}_{w}\right)\right|_{w=0}=-\alpha<0
$$

and then, by Lemma 3.3, we conclude.

As a consequence of Proposition 3.4 we immediately get the following fundamental properties of the relations $\ll$ and $\leq$ (compare also with [35, Corollary1]):

Corollary 3.5. Let $p, q, z \in \tilde{M}$. If $p \leq q$ and $q \ll z$ (or vice versa) then $p \ll z$.

Moreover, the equality between the closures of the chronological and the causal future of a point also easily follows:

Corollary 3.6. For all $p \in \tilde{M}, \overline{J^{+}(p)}=\overline{I^{+}(p)}$ (and $\overline{J^{-}(p)}=\overline{I^{-}(p)}$ ).

Proof. From (6), it is enough to show that $J^{+}(p) \subset \overline{I^{+}(p)}$. Let $q=(t, x) \in J^{+}(p)$. Clearly, if $q=p$ then $q \in \overline{I^{+}(p)}$. So, let us assume that $p<q$; let $\gamma$ be a causal future-pointing curve between $p$ and $q$ and and consider a sequence of points $q_{k}=\left(t_{k}, x\right)$, with $t_{k} \searrow t$. As the line $l_{x}$ is timelike, from Corollary 3.5 , we get $p \ll q_{k}$, hence $q \in \overline{I^{+}(p)}$.

Let us now give a first definition in the causal hierarchy of Finsler spacetimes which, as the following ones in Definitions 3.9 and 3.14 are formally the same as in Lorentzian spacetimes. 
Definition 3.7. A Finsler spacetime $(\tilde{M}, \tilde{g})$ is causally simple if for all $p \in \tilde{M}$, $J^{ \pm}(p)$ are closed and no closed future-pointing causal curve exists.

The following result extends to standard static Finsler spacetimes [14, Th. 4.3(a)].

Theorem 3.8. A standard static Finsler spacetime $(\tilde{M}, L)$ is causally simple if and only if for any couple $(x, y) \in M \times M$ there exists a geodesic of the metric $\tilde{F}=F / \sqrt{\Lambda}$ joining $x$ to $y$ with length equal to the their $\tilde{F}$-distance, $\tilde{d}(x, y)$.

Proof. $(\Rightarrow)$ Let $x, y \in M$ and let $\sigma:[0,1] \rightarrow M$ be a piecewise smooth curve curve, say, from $x$ to $y$. The curve $\gamma(s)=\left(\int_{0}^{s} \tilde{F}(\dot{\sigma}) d \mu, \sigma(s)\right)$ is then lightlike and future-pointing. This shows that $J^{+}(0, x) \cap l_{y} \neq \emptyset$. Let $t_{y}:=\inf \{t \in \mathbb{R}$ : $\left.(t, y) \in J^{+}(0, x)\right\} ;$ clearly, $\left(t_{y}, y\right) \in \overline{J^{+}(0, x)}=J^{+}(0, x)$. Moreover $\left(t_{y}, y\right) \notin I^{+}(0, x)$ otherwise, being $I^{+}(0, x)$ open there would exist $(t, y) \in I^{+}(0, x)$ with $t<t_{y}$. Thus, from Proposition 3.4, any causal future-pointing curve connecting $(0, x)$ to $\left(t_{y}, y\right)$ must be a lightlike geodesic and, from Proposition 2.18, its component on $M$ is a pregeodesic of $(M, \tilde{F})$. Moreover, the $(\tilde{F})$-length of this component must be equal to $\tilde{d}(x, y)$ otherwise it would be possible to consider, as above, a lightlike future-pointing curve whose future endpoint would have $t$-coordinate less than $t_{y}$. $(\Leftarrow)$ Let $\left(t_{0}, x_{0}\right) \in \tilde{M}$ and $(t, x) \in \overline{J^{+}\left(t_{0}, x_{0}\right)}$. Consider a geodesic $\sigma:[0,1] \rightarrow M$ connecting $x_{0}$ to $x$ and such that $\ell(\sigma)=\tilde{d}\left(x_{0}, x\right)$. Then, the curve $\gamma(s)=$ $\left(t_{0}+\int_{0}^{s} \tilde{F}(\dot{\sigma}) d \mu, \sigma(s)\right)$ is lightlike and future-pointing. Let $\left\{\left(t_{k}, x_{k}\right)\right\}$ be a sequence of points such that $\left(t_{k}, x_{k}\right) \in J^{+}\left(t_{0}, x_{0}\right)$ and $\left(t_{k}, x_{k}\right) \rightarrow(t, x)$, as $k \rightarrow+\infty$. Consider a sequence of future-pointing causal curves $\gamma_{k}$, each one connecting $\left(t_{0}, x_{0}\right)$ to $\left(t_{k}, x_{k}\right)$, so that $\tilde{d}\left(x_{0}, x_{k}\right) \leq t_{k}-t_{0}$. Then, by the continuity of $\tilde{d}$, we get $\tilde{d}\left(x_{0}, x\right) \leq t-t_{0}$. Thus, if $\tilde{d}\left(x_{0}, x\right)<t-t_{0}$ then, by $(7),\left(t_{0}, x_{0}\right) \ll(t, x)$ and, if $\tilde{d}\left(x_{0}, x\right)=t-t_{0}$ the same lightlike curve $\gamma$ gives $\left(t_{0}, x_{0}\right) \leq(t, x)$.

Since the Finslerian distance $\tilde{d}$ of $\tilde{F}$ is not symmetric, the lack of symmetry gives rise to two notions of completeness: the forward completeness and the backward one (see [5, §6.2 D]). A forward (resp. backward) Cauchy sequence in a Finsler manifold $(M, F)$ is a sequence $\left\{x_{k}\right\}_{k \in \mathbb{N}}$ with the property that for all $\varepsilon>0$ there exists $\nu \in \mathbb{N}$ such that for all $k>j \geq \nu, d\left(x_{j}, x_{k}\right)<\varepsilon$ (resp. $\left.d\left(x_{k}, x_{j}\right)<\varepsilon\right)$, where $d$ is the distance associated to $F$. Then $(M, F)$ is said forward (resp. backward) complete if any forward (resp. backward) Cauchy sequence is convergent.

Forward and backward completeness of the metric $\tilde{F}$ are related to the existence of some particular Cauchy hypersurfaces in $(\tilde{M}, L)$.

Definition 3.9. A Cauchy hypersurface in a Finsler spacetime $(\tilde{M}, \tilde{g})$ is a topological hypersurface that is met exactly once by every inextensible future-pointing causal curve.

The following theorem extends to standard static Finsler spacetime a well known result, already cited at the beginning of Section 2, valid for standard static Lorentzian spacetimes, and [14, Theorem 4.4] for standard stationary ones.

Theorem 3.10. A slice (and then any slice) $S_{t}=\{t\} \times M$ is a Cauchy hypersurface of the standard static Finsler spacetime $(\tilde{M}, L)$ if and only the Finsler manifold $(M, \tilde{F})$ is forward and backward complete. 
Before proving Theorem 3.10 we need the following well known result that we report for the reader convenience.

Lemma 3.11. Let $(M, F)$ be a Finsler manifold. Then $(M, F)$ is forward (resp. backward) complete if and only if for any piecewise smooth curve of finite Finslerian length $\sigma:[a, b) \rightarrow M$ (resp. $\sigma:(a, b] \rightarrow M)$ there exists a point $x_{0} \in M$ such that $\sigma(s) \rightarrow x_{0}$, as $s \rightarrow b$ (resp. as $\left.s \rightarrow a\right)$.

Proof. Let us show the equivalence only for forward completeness since the backward case is completely analogous.

$\Rightarrow)$ Let $\ell(\sigma) \in(0,+\infty)$ be the length of $\sigma$. Set $K=\{x \in M: d(\sigma(a), x) \leq \ell(\sigma)\}$. Since $K$ is forward bounded and closed, we have that it is compact by Hopf-Rinow theorem (see [5, Th.6.6.1]). Fix a sequence $\left\{s_{k}\right\}$ in $[a, b)$ such that $s_{k} \rightarrow b$. Since $\sigma([a, b)) \subseteq K$, the compactness implies that there exists a point $x_{0} \in K$ such that $\sigma\left(s_{k}\right)$ converges to $x_{0}$, up to subsequences. If $\lim _{s \rightarrow b} \sigma(s) \neq x_{0}$, there would exist an $\varepsilon>0$ such that $\sigma$ leaves the set $\left\{x \in M: d\left(x, x_{0}\right) \leq \varepsilon\right\}$ infinite times while, definitively, $d\left(\sigma\left(s_{k}\right), x_{0}\right)<\varepsilon / 2$. This is a contradiction because $\sigma$ has a finite length. $(\Leftarrow)$ We suppose that $(M, F)$ is not forward complete manifold. Therefore, there exists a geodesic $\sigma:[a, b) \rightarrow M$, hence with finite length, which is not extendible to $s=b$.

Proof of Theorem 3.10. $(\Rightarrow)$ By contradiction, assume that $(M, \tilde{F})$ is not, say, forward complete. Then, from Lemma 3.11, there exists $\sigma:[a, b) \rightarrow M$ having finite length, $\tilde{\ell}(\sigma)=\int_{a}^{b} \tilde{F}(\dot{\sigma}) d s$, and such that it does not converge as $s \rightarrow b$. Take $t_{0} \in \mathbb{R}$ such that $t_{0}+\ell(\sigma)<t$ and define $\theta:[a, b) \rightarrow \mathbb{R}, \theta(s)=t_{0}+\tilde{\ell}\left(\left.\sigma\right|_{[a, s]}\right)$ and $\gamma:[a, b) \rightarrow \mathbb{R} \times M, \gamma(s)=(\theta(s), \sigma(s))$. Observe that $\gamma$ is a future-pointing lightlike curve, by definition, and it is future-inextensible because $\sigma$ is inextensible. Hence, any extension of $\gamma$ as a past inextensible causal curve does not intersect $S_{t}$, a contradiction.

$(\Leftarrow)$ Let $\left(t_{0}, x_{0}\right) \in \mathbb{R} \times M$, with $t_{0}<t$. We consider a future-inextensible causal curve $\gamma:[a, b) \rightarrow \mathbb{R} \times M, \gamma(s)=(\theta(s), \sigma(s))$ that starts from $\left(t_{0}, x_{0}\right)$. As $\theta$ is an increasing function, $\gamma$ cannot intersect $S_{t}$ more than once. So assume, by contradiction, that $\gamma$ does not meet $S_{t}$ then, as $\theta$ is also continuous, $\lim _{s \rightarrow b} \theta(s) \in$ $\left(t_{0}, t\right]$. Moreover, being $\gamma$ causal, $\int_{a}^{s} \tilde{F}(\dot{\sigma}) d \mu \leq \theta(s)-t_{0}$, for all $s \in[a, b)$. Hence, there exists $\lim _{s \rightarrow b^{-}} \int_{a}^{s} \tilde{F}(\dot{\sigma}) d \mu \in\left[0, t-t_{0}\right]$, i.e $\sigma$ has a finite length and therefore from Lemma 3.11 it is extendible. Hence, $\gamma$ is future extendible. The proof for a past-inextensible curve is analogous.

Notice that, as $L$ and $\tilde{L}$ share the same causal curves, $S_{t}=\{t\} \times M$ is a Cauchy hypersurface also for $(\tilde{M}, \tilde{L})$. Anyway, the completeness assumptions in Theorem 3.10 involve the optical metric $\tilde{F}$ and not $F$. We can give some conditions on $\Lambda$ ensuring forward and backward completeness of the conformal metric $\tilde{F}=$ $F / \sqrt{\Lambda}$, provided that $F$ is forward and backward complete. To this end, let us introduce the following notions.

Given a Finsler manifold $(M, F)$ and a function $\Lambda: M \rightarrow \mathbb{R}$, we say that $\Lambda$ is forward (resp. backward) subquadratic, if there exist constants $c_{1}, c_{2}>0$ such that, for all $x \in M,|\Lambda(x)| \leq c_{1} d(\bar{x}, x)^{2}+c_{2}\left(|\Lambda(x)| \leq c_{1} d(x, \bar{x})^{2}+c_{2}\right)$ for some $\bar{x} \in M$. Clearly, by the triangle inequality, one immediately sees that previous definitions are independent from the point $\bar{x}$ (up to change the constants $c_{1}$ and $c_{2}$ ). Moreover, we say that $\Lambda$ is subquadratic if it is both forward and backward subquadratic. 
Proposition 3.12. Let $(M, F)$ be a forward (resp. backward) complete Finsler manifold. If $\Lambda$ is a positive smooth forward (resp. backward) subquadratic function then $(M, \tilde{F})$ is forward (resp. backward) complete.

Proof. Let $\left\{x_{k}\right\}_{k \in \mathbb{N}}$ be a forward Cauchy sequence for $(M, \tilde{F})$ and let us denote by $\tilde{d}$ the distance induced by $\tilde{F}$. We can find two indexes $\nu$ and $\bar{m}$ such that, for all $k>\bar{m}>\nu, \tilde{d}\left(x_{\bar{m}}, x_{k}\right)<\frac{1}{2 \sqrt{c_{1}}}$. Let $\sigma_{k}:[0,1] \rightarrow M$ be a curve joining $x_{\bar{m}}$ to $x_{k}$ and such that

$$
\int_{0}^{1} \tilde{F}\left(\dot{\sigma}_{k}\right) d s<\frac{1}{2 \sqrt{c_{1}}} .
$$

Now we evaluate the $F$-distance $d\left(x_{\bar{m}}, x_{k}\right)$ :

$$
\begin{aligned}
\int_{0}^{1} F\left(\dot{\sigma}_{k}\right) d s & =\int_{0}^{1} \frac{1}{\sqrt{\Lambda\left(\sigma_{k}\right)}} F\left(\dot{\sigma}_{k}\right) \sqrt{\Lambda\left(\sigma_{k}\right)} d s \\
& \leq \int_{0}^{1} \tilde{F}\left(\dot{\sigma}_{k}\right)\left(c_{1} d\left(\bar{x}, \sigma_{k}\right)^{2}+c_{2}\right)^{\frac{1}{2}} d s \\
& \leq \int_{0}^{1} \tilde{F}\left(\dot{\sigma}_{k}\right)\left(c_{1}\left(2 d\left(x_{\bar{m}}, \sigma_{k}\right)^{2}+2 d\left(\bar{x}, x_{\bar{m}}\right)^{2}\right)+c_{2}\right)^{\frac{1}{2}} d s \\
& \leq \int_{0}^{1} \tilde{F}\left(\dot{\sigma}_{k}\right)\left(2 c_{1}\left(\int_{0}^{s} F\left(\dot{\sigma}_{k}\right) d \mu\right)^{2}+2 c_{1} d\left(\bar{x}, x_{\bar{m}}\right)^{2}+c_{2}\right)^{\frac{1}{2}} d s \\
& <\frac{1}{\sqrt{2}} \int_{0}^{1} F\left(\dot{\sigma}_{k}\right) d s\left(1+\frac{2 c_{1} d\left(\bar{x}, x_{\bar{m}}\right)^{2}+c_{2}}{2 c_{1} \int_{0}^{1} F\left(\dot{\sigma}_{k}\right) d s}\right)^{\frac{1}{2}} d s .
\end{aligned}
$$

This shows that the sequence $\left\{\int_{0}^{1} F\left(\dot{\sigma}_{k}\right) d s\right\}_{m \in \mathbb{N}}$ is bounded. Thus $\left\{x_{k}\right\}$ is forward bounded with respect to $d$ and therefore it admits a converging subsequence. The proof in the backward case is analogous.

From the above proposition and Theorem 3.10 we get:

Corollary 3.13. If $(M, F)$ is forward and backward complete and $\Lambda$ is subquadratic, then $S_{t}=\{t\} \times M$ is a Cauchy hypersurface in $(\tilde{M}, L)$.

The strongest property in the causal hierarchy of spacetimes is global hyperbolicity:

Definition 3.14. A Finsler spacetime $(\tilde{M}, \tilde{g})$ is globally hyperbolic if it is causal (i.e. no future-pointing closed causal curve exists) and $J^{+}(p) \cap J^{-}(q)$ is a compact subset, for every $p, q \in \tilde{M}$.

Classically, a spacetime is globally hyperbolic if it is strongly causal (namely, no almost closed future-pointing causal curve exists). As shown recently in [11], the weaker property of being causal can be used instead. Anyway, in a standard static Finsler spacetime both conditions hold because every future-pointing causal curve has increasing $t$-component. The following theorem is the analogous of [14, Th. 4.3-(b)] which concerns standard stationary Lorentzian spacetimes.

Theorem 3.15. A standard static Finsler spacetime $(\tilde{M}, L)$ is globally hyperbolic if and only if $\bar{B}^{+}(x, r) \cap \bar{B}^{-}(y, s)$ is compact, for every $x, y \in M$ and $r, s>0$, where $\bar{B}^{ \pm}$are the the closure of the forward and backward balls on $M$ w.r.t. to the distance $\tilde{d}$ associated to $F / \sqrt{\Lambda}$. 
Proof. $(\Rightarrow)$ Let us first show that $J^{+}\left(t_{0}, x_{0}\right)$ is closed for all $\left(t_{0}, x_{0}\right) \in \tilde{M}$. Let $(t, x) \in \overline{J^{+}\left(t_{0}, x_{0}\right)}$. Clearly we can find $\left(t_{1}, x_{1}\right)$ such that $(t, x) \in \overline{J^{+}\left(t_{0}, x_{0}\right)} \cap$ $I^{-}\left(t_{1}, x_{1}\right)$. Then, being $I^{-}\left(t_{1}, x_{1}\right)$ open, any sequence $\left\{\left(t_{k}, x_{k}\right)\right\} \subset J^{+}\left(t_{0}, x_{0}\right)$ and converging to $(t, x)$ is definitively contained in $I^{-}\left(t_{1}, x_{1}\right)$ and admits, by global hyperbolicity, a converging subsequence to a point $(\tilde{t}, \tilde{x}) \in J^{+}\left(t_{0}, x_{0}\right) \cap J^{-}\left(t_{1}, x_{1}\right)$. As $(\tilde{t}, \tilde{x})$ must be equal to $(t, x)$, it follows that $J^{+}\left(t_{0}, x_{0}\right)$ is closed. Analogously, the same holds for $J^{-}\left(t_{0}, x_{0}\right)$. Thus, for any $(r, x) \in \tilde{M}$, by Proposition 3.2 and Corollary 3.6, we get

$$
\begin{aligned}
\{0\} \times\left(\bar{B}^{+}(x, r) \cap \bar{B}^{-}(x, r)\right)= & \\
\overline{I^{+}(-r, x)} \cap \overline{I^{-}(r, x)} & =\overline{J^{+}(-r, x)} \cap \overline{J^{-}(r, x)}=J^{+}(-r, x) \cap J^{-}(r, x) .
\end{aligned}
$$

Hence, the left-hand side is a compact set and this can be easily seen to be equivalent to the thesis (see, e.g. [14, Prop. 2.2]).

$(\Leftarrow)$ For every $\left(t_{0}, x_{0}\right),\left(t_{1}, x_{1}\right) \in \tilde{M}$, by Proposition 3.2 and Corollary 3.6, we get:

$$
\overline{J^{+}\left(t_{0}, x_{0}\right)} \cap \overline{J^{-}\left(t_{1}, x_{1}\right)}=\bigcup_{s \in\left[0, t_{1}-t_{0}\right]}\left\{t_{0}+s\right\} \times\left(\bar{B}^{+}\left(x_{0}, s\right) \cap \bar{B}^{-}\left(x_{1}, t_{1}-t_{0}-s\right)\right) .
$$

As in [14, p. 936], one obtains that the right-hand side is a compact subset and, then, $\overline{J^{+}\left(t_{0}, x_{0}\right)} \cap \overline{J^{-}\left(t_{1}, x_{1}\right)}$ is compact. From [14, Prop. 2.2 and Th. 5.2], we know that the compactness of $\bar{B}^{+}(x, r) \cap \bar{B}^{-}(y, s)$, for any $x, y \in M$ and $r, s>0$, implies that any pair of points $x, y \in M$ can be joined by a geodesic from $x$ to $y$ with length $\tilde{d}(x, y)$. Hence, from Theorem 3.8 , both $J^{+}\left(t_{0}, x_{0}\right)$ and $J^{-}\left(t_{1}, x_{1}\right)$ are closed and this concludes the proof.

Remark 3.16. Notice that, in the proof of Theorem 3.15 we have also shown that global hyperbolicity implies causal simplicity as in the causal ladder for Lorentzian spacetime. From the Finslerian Hopf-Rinow theorem (see [5, Th.6.6.1]), it is clear that forward and backward completeness of the metric $\tilde{F}$ implies compactness of the intersections of the closed balls (in other words, the fact that a slices $S_{t}$ is a Cauchy hypersurface implies that $(\tilde{M}, L)$ is globally hyperbolic). Anyway, the latter condition is weaker than forward or backward completeness (see [14, Example 4.6]), so a standard static Finsler spacetime might be globally hyperbolic but with slices $S_{t}$ which are not Cauchy hypersurface. It is well known that in a Lorentzian spacetime $\tilde{M}$, global hyperbolicity is equivalent to the existence of a smooth, spacelike Cauchy hypersurface [9] and a Cauchy temporal function [10], i.e. a smooth function $f: \tilde{M} \rightarrow \mathbb{R}$ which is strictly increasing on any future-pointing causal curve and whose level sets are (spacelike, smooth) Cauchy hypersurfaces. As observed in [24, Theorem 1], the existence of a Cauchy temporal function follows also in a Finsler spacetime by using weak KAM theory as shown in [19]. Indeed, [19, Th. 1.3] extends the result in [10] to a manifold $\tilde{M}$ endowed with a cone structure $\mathcal{C}$, i.e. a continuous map (w.r.t. the Hausdorff metric in local coordinates) $p \in \tilde{M} \mapsto \mathcal{C}_{p} \subset T_{x} \tilde{M}$, where $\mathcal{C}_{p}$ is a closed convex cone with vertex at 0 , with non-empty interior and not containing any complete affine line. Clearly, from Lemma 2.21, the set of future-pointing causal vectors plus the zero section in a standard static Finsler spacetime defines a cone structure. It is however worth pointing out that, when applied to Finsler spacetimes, [19, Th. 1.3] does not automatically gives a spacelike hypersurface (in the sense of Definition 1.6). This is due to the fact that a cone structure takes into account only future-pointing directions 
and the cones might flatten "at infinity", i.e. along some lines corresponding to future-pointing lightlike vectors at the points outside an arbitrarily large compact subset of $\tilde{M}$. Thus, in principle, the tangent bundle of a level set of a temporal function might contain vectors which are causal and past-pointing.

For this reason, we introduce the following:

Definition 3.17. Let $(\tilde{M}, L)$ be a Finsler spacetime. A hypersurface $\mathcal{H} \subset \tilde{M}$ is said future spacelike if $T \mathcal{H}$ does not contain any future-pointing causal vector.

We can now show that a future Cauchy hypersurface can be always constructed as the graph of a smooth function on $M$ (as for a standard stationary Lorentzian spacetime, see the proof of [14, Th. 5.10]).

Proposition 3.18. Let $(\tilde{M}, L)$ be a globally hyperbolic standard static Finsler spacetime such that $\tilde{F}$ is not forward or backward complete. Then there exists a smooth function $f: M \rightarrow \mathbb{R}$ such that $S_{f}=\{(f(x), x): x \in M\}$ is a future spacelike, smooth, Cauchy hypersurface.

Proof. As $(\tilde{M}, L)$ is globally hyperbolic, from Theorem 3.15, the sets $\bar{B}^{+}(x, r) \cap$ $\bar{B}^{-}(x, r)$ are compact for any $x \in M$ and $r>0$. Then from [32, Th. 1], there exists a smooth function $f: M \rightarrow \mathbb{R}$ such that $\tilde{F}-d f$ is a backward and forward complete Finsler metric on $M$ (in particular, $\tilde{F}(v)-d f(v)>0$ for all $v \in T M \backslash 0$ ). Notice that for all $\tilde{v} \in T S_{f}, \tilde{v}=(d f(v), v), v \in T M$, we have $L(\tilde{v})=-\Lambda(x)(d f(v))^{2}+F^{2}(v)$, where $x=\tilde{\pi}(\tilde{v})$. Being $\tilde{F}(v)-d f(v)>0$ for all $v \in T M \backslash 0$, we conclude that $L((d f(v), v))>0$ for all $v$ such that $d f(v)>0$ or, equivalently, that $S_{f}$ is a future spacelike hypersurface. Let $\gamma:(a, b) \rightarrow \tilde{M}, \gamma(s)=(\theta(s), \sigma(s))$, be an inextensible causal curve and assume that $\gamma$ intersects $S_{f}$ at least twice at the instants $s_{1}, s_{2} \in$ $(a, b), s_{1}<s_{2}$. Thus, we have

$$
0=\int_{s_{1}}^{s_{2}}(\dot{\theta}-d f(\dot{\sigma})) d s \geq \int_{s_{1}}^{s_{2}}(\tilde{F}(\dot{\sigma})-d f(\dot{\sigma})) d s,
$$

which is possible if and only if $\sigma$ is constant on $\left[s_{1}, s_{2}\right]$ but this is a contradiction because $S_{f}$ is the graph of $f$. Let now assume that $\gamma$ does not intersect $S_{f}$. Then, being $\theta$ and $\sigma$ continuous, it must be either $\theta-f \circ \sigma<0$ or $\theta-f \circ \sigma>0$ on $(a, b)$. Assume that the former inequality holds and take $s_{0} \in(a, b)$. We have, for any $s>s_{0}$,

$$
\int_{s_{0}}^{s} \tilde{F}(\dot{\sigma})-d f(\dot{\sigma}) d \mu \leq \theta(s)-\theta\left(s_{0}\right)-f(\sigma(s))+f\left(\sigma\left(s_{0}\right)\right)<f\left(\sigma\left(s_{0}\right)\right)-\theta\left(s_{0}\right) .
$$

Therefore $\left.\sigma\right|_{\left[s_{0}, b\right)}$ has finite length w.r.t. $\tilde{F}-d f$. As this metric is forward complete, from Lemma 3.11, $\sigma$ is extendible in $b$, i.e. there exist $x_{b} \in M$ such that $x_{b}=$ $\lim _{s \rightarrow b} \sigma(s)$. So, by continuity of $f$ and monotonicity of $\theta$ also $\theta$ is extendible in $b$, which contradicts the fact that $\gamma$ was future inextensible. By a similar reasoning, using that $\tilde{F}-d f$ is also backward complete and $\gamma$ is past inextensible, we obtain that also the second inequality cannot hold.

The following proposition shows that, under the condition of finite reversibility (10) (reversibility is a measure of how much a Finsler metric is far from being reversible; it was introduced in [41]), it is possible to modify $f$ to get a smooth, spacelike (not only future spacelike), Cauchy hypersurface. 
Proposition 3.19. Under the assumptions and notations of Proposition 3.18, assume also that

$$
\alpha:=\sup _{v \in T M \backslash 0} \frac{F(v)}{F(-v)}<+\infty,
$$

then $S_{f / \alpha}$ is a spacelike Cauchy hypersurface.

Proof. We show that, under (10), both $\tilde{F}-d f / \alpha$ and $\tilde{F}^{-}-d f / \alpha$, are Finsler metric with the former which is also forward and backward complete, where $\tilde{F}^{-}$denotes the reverse Finsler metric associated to $\tilde{F}, \tilde{F}^{-}(v):=\tilde{F}(-v)$, and $f$ is a function such that $\tilde{F}-d f$ is a forward and backward complete Finsler metric, [32, Th. 1]. Observe that $\alpha \geq 1$ and it is equal to 1 if and only if $F$ is a reversible Finsler metric, i.e. $F(v)=F(-v)$ for all $v \in T M$. Clearly, we have $\tilde{F}(v)-\frac{d f(v)}{\alpha}>0$, for all $v \in T M \backslash 0$. Moreover, for all $v \in T M \backslash 0$ with $d f(v) \geq 0$, we have $\frac{d f(v)}{\alpha} \leq \frac{d f(v) F(-v)}{F(v)}=\frac{d f(v) \tilde{F}(-v)}{\tilde{F}(v)}<\tilde{F}(-v)=\tilde{F}^{-}(v)$. On the other hand, if $d f(v)<0$ then $\tilde{F}^{-}(v)-\frac{d f(v)}{\alpha}>0$. Hence, both $\tilde{F}-d f / \alpha$ and $\tilde{F}^{-}-d f / \alpha$, are Finsler metric (see, e.g., [23, Cor. 4.17]). Clearly, $\tilde{F}-d f / \alpha$ remains backward and forward complete because it is $1 / \alpha$-homothetic, to $\alpha \tilde{F}-d f$. Thus the same proof of Proposition 3.18 shows that $S_{f / \alpha}$ is a future spacelike Cauchy hypersurface. Actually, for $d f(v)<0$, we also have $\tilde{F}^{-}(-v)>-\frac{d f(v)}{\alpha}$, hence $-\tilde{F}(v)<\frac{d f(v)}{\alpha}$ which is equivalent, together with $\tilde{F}(v)>\frac{d f(v)}{\alpha}$, to $F^{2}(v)-\Lambda(x) \frac{(d f(v))^{2}}{\alpha^{2}}>0$, for all $v \in T_{x} M$ and $x \in M$. Since, $T S_{f / \alpha}=\left\{\left(\frac{d f(v)}{\alpha}, v\right): v \in T M\right\}$ and $L\left(\left(\frac{d f(v)}{\alpha}, v\right)\right)=F^{2}(v)-\Lambda(x) \frac{(d f(v))^{2}}{\alpha^{2}}$, we have done.

We have seen in Theorem 3.8 that causal simplicity is equivalent to the existence of a $\tilde{F}$-length minimizing geodesic between any couple of points $(x, y) \in M \times M$ and then from Proposition 2.18 and Remark 2.19 to the existence of a future-pointing lightlike geodesic between, $\left(t_{0}, x\right)$ and $l_{y}$ (for any $t_{0} \in \mathbb{R}$ ) which minimizes the arrival time functional $T_{\left(t_{0}, x\right) l_{y}}$. Under non-trivial topology of the manifold $M$ we can obtain also a multiplicity result.

Corollary 3.20. Let $(\tilde{M}, L)$ be a globally hyperbolic standard static Finsler spacetime such that $M$ is not contractible. Then for any $p, q \in \tilde{M}$ there exist infinitely many lightlike future-pointing geodesics $\left\{\gamma_{k}\right\}_{k \in \mathbb{N}}$ connecting $p$ to $l_{x_{q}}$ and such that $T_{p l_{x_{q}}}\left(\gamma_{k}\right) \rightarrow+\infty$, as $k \rightarrow+\infty$.

Proof. From global hyperbolicity, $\bar{B}^{+}(x, r) \cap \bar{B}^{-}(y, s)$ is compact for any $x, y \in M$ and $r, s>0$. Then from [14, Th. 5.2] we know that there exists a sequence $\left\{\sigma_{k}\right\}$ of geodesics for $\tilde{F}$ from $x_{p}$ to $x_{q}$ such that $\tilde{\ell}\left(\sigma_{k}\right) \rightarrow+\infty$. Thus, it is enough to apply Proposition 2.18 and Remark 2.19.

Remark 3.21. The above corollary is based on Lüsternik-Schnirelmann theory for Finsler geodesics as developed in [13]. Indeed the fact that $M$ is not contractible implies that the Lüsternik-Schnirelmann category of the based loop space is equal to $+\infty$ and compact subsets of it, with arbitrarily large category, do exist [18]. Similar results concerning existence and multiplicity of geodesics of $(\tilde{M}, L)$ between two given events can be obtained by matching the results in [31] and the ones in [13]. 


\section{Conclusions And FURTher Discussions}

We have extended the class of standard static Lorentzian spacetimes $\mathbb{R} \times M$ by considering Finslerian optical metrics. A spherical symmetric Finsler optical metric has been proposed in [28] to get a perturbation of the Schwarzschild metric. As observed in [28], the most important feature that distinguishes a Finsler metric from a Lorentzian one is the fact that it breaks spacetime isotropy also at an infinitesimal scale. In the case of a standard static Finsler spacetime, the anisotropy is confined to the restspaces $\{t\} \times M$ (where $t$ is the natural coordinate on $\mathbb{R}$ ), relative to the observer field $\partial_{t}$, through the optical metric $F / \Lambda$. We also observe that the map $(t, x) \mapsto(-t, \varphi(x))$ of $\tilde{M}$, where $\varphi$ is a diffeomorphism of $M$, flips the future causal cone in the past one at $T_{(-t, \varphi(x))} \tilde{M}$. If we assume that $\Lambda$ is constant and $F$ is a locally Minkowski, non-reversible, metric on $M$ (i.e. there exists a covering of $M$ by coordinate systems such that, in the corresponding natural coordinates $\left(x^{1}, \ldots, x^{n}, v^{1}, \ldots, v^{n}\right)$ of $T M, F$ depends only on $\left.\left(v^{i}\right)\right)$ then the causal structure of $\tilde{M}$ is not invariant under the $P T$-transformation $\left(\tau, v^{1}, \ldots, v^{n}\right) \mapsto$ $\left(-\tau,-v^{1}, \ldots,-v^{n}\right)$. So such a particular class of standard static Finsler spacetimes could be interesting for some extensions of the Standard Model of particles when CPT symmetry and Lorentz-invariance violation are considered $[43,17]$.

A wider class of splitting Finsler spacetimes $\tilde{M}=\mathbb{R} \times M$ can be obtained, taking a one-form $\omega$ on $M$ and a function $\Lambda: M \rightarrow \mathbb{R}$ which is non-necessarily positive, by considering the quadratic Finsler function $L: T \tilde{M} \rightarrow \mathbb{R}$,

$$
L((\tau, v))=-\Lambda \tau^{2}+2 \tau \omega(v)+F^{2}(v) .
$$

It can be easily seen (compare with [15, Prop. 3.3]) that the fundamental tensor $\tilde{g}=-\Lambda d t^{2}+\omega \otimes d t+d t \otimes \omega+g$ of $L$, where $g$ is the fundamental tensor of $F$, is a smooth, symmetric section of index 1 of the tensor bundle $\pi^{*}(T \tilde{M}) \otimes \pi^{*}(T \tilde{M})$ over $T \tilde{M} \backslash \mathcal{T}$, where $\mathcal{T}$ is again the line bundle defined by $\partial_{t}$, if and only if $\Lambda(x)+\|\omega\|_{x}>0$ for all $x \in M$, where

$$
\|\omega\|_{x}=\min _{v \in T_{x} M \backslash\{0\}} \max _{w \in T_{x} M \backslash\{0\}} \frac{\left|\omega_{x}(w)\right|}{\sqrt{g_{v}(w, w)}},
$$

(so, in particular, if $\Lambda$ is a positive function). The vector field $\partial_{t}$ is still a Killing vector field but it is no more timelike (it is spacelike at the points $(t, x)$ where $\Lambda(x)<0$ and lightlike iff $\Lambda(x)=0)$ and no more $\tilde{g}_{(\tau, v)}$-orthogonal to the vectors in $\{0\} \times T_{x} M, x=\pi^{M}(v)$ (recall Remark 2.10). On the other hand, the function $t: \tilde{M} \rightarrow \mathbb{R}$ is a temporal function in the sense that it is smooth and $d t(\tilde{v}) \neq 0$ for any causal vector $\tilde{v} \in T \tilde{M}$. This allows us to define future-pointing causal vectors as the ones such that $d t(\tilde{v})>0$. So, for a function $\Lambda$ which is not positive our definition of a Finsler spacetime (Definition 1.1) should be now intended in the weaker sense of Remark 1.3: $\tilde{g}_{\tilde{v}}(\tilde{v}, \tilde{v})$ is positive in a punctured neighbourhood of $\mathcal{T}_{p}$, for any $p=(t, x) \in \tilde{M}$ such that $\Lambda(x)>0$, while there always exist vectors $\tilde{v}_{1}, \tilde{v}_{2}$, in any punctured neighbourhood of $\mathcal{T}_{(t, x)}$, such that $\tilde{g}_{\tilde{v}_{1}}\left(\tilde{v}_{1}, \tilde{v}_{1}\right)>0$ and $\tilde{g}_{\tilde{v}_{2}}\left(\tilde{v}_{2}, \tilde{v}_{2}\right)<0$, iff $\Lambda(x)=0$. In the case where $\Lambda$ is positive, such Finsler spacetimes come into play: (a) already when one consider a different splitting of the type $\mathbb{R} \times S_{f}$, in standard static Finsler spacetime, after a coordinate change of the type $(t, x) \mapsto(t+f(x), x)$ (recall Proposition 3.19); (b) as a Finslerian generalization of a standard stationary Lorentzian spacetime where the Riemannian metric $g_{0}$ is replaced by the fundamental tensor $g$ of the Finsler metric $F$. In the general 
case where $\Lambda$ is not positive, they represent the spacetime counterpart of wind Finslerian structures morally in the same way as SSTK splittings correspond to wind Riemannian structures (see [15]) and they certainly deserve further study. In particular, a challenging task is the study of the relations between their causality properties and the geometry of their optical structure (associated to future-pointing lightlike vectors). The latter is described, in the general case of a non-positive $\Lambda$, by two conic pseudo-Finsler metrics (in the sense of [23]):

$$
\begin{aligned}
& F^{o}(v)=\frac{F^{2}(v)}{-\omega(v)+\sqrt{\Lambda F^{2}(v)+\omega(v)^{2}}}, \\
& F_{l}^{o}(v)=-\frac{F^{2}(v)}{\omega(v)+\sqrt{\Lambda F^{2}(v)+\omega(v)^{2}}},
\end{aligned}
$$

The cone structure $\mathcal{A} \subset T M$, where the first metric is defined, is given by the whole tangent space $T_{x} M$, for all $x \in M$ with $\Lambda(x)>0$, by the vectors $v$ such that $\omega_{x}(v)<0$ plus 0 at the points where $\Lambda(x)=0$ and by the vectors $\{v \in$ $\left.T M_{l}: \omega(v)<0, \Lambda F^{2}(v)+\omega(v)^{2} \geq 0\right\}$ on $M_{l}$. This last cone structure is also the set where $F_{l}^{0}$ is defined and we have, there, $0<F^{o}(v) \leq F_{l}^{o}(v)$, with equality if and only if $\Lambda F^{2}(v)+\omega(v)^{2}=0$. This is the condition that the projections of the lightlike vectors of $(\tilde{M}, L)$ on $T M_{l}$ must satisfy. The break of continuity (see [19, Def. 2.25]) of the map $x \in M \mapsto \mathcal{A}_{x}$ at the critical region $\{x \in M: \Lambda(x)=0\}$ is

certainly a difficulty in dealing with such cone structures but the spacetime point of view, as shown in [15], is of help since the map that to $p \in \tilde{M}$ associates the set of future-pointing causal vectors at $p$ is instead continuous.

\section{ACKNOWLEDGEMENTS}

We would like to thank the referee for his stimulating comments and for pointing out some references. We also thank some interesting comments and remarks by M. A. Javaloyes and M. Sánchez.

EC is a member of and has been partially supported during this research by the "Gruppo Nazionale per l'Analisi Matematica, la Probabilità e le loro Applicazioni" (GNAMPA) of the "Istituto Nazionale di Alta Matematica (INdAM)", by "FRA2011, Politecnico di Bari", and by the project MTM2013- 47828-C2-1-P (Spanish MINECO with FEDER funds).

GS is a member of the "Gruppo Nazionale per le Strutture Algebriche Geometriche e loro Applicazioni" (GNSAGA).

\section{REFERENCES}

[1] A.B. Aazami, M.A., Javaloyes, Penrose's singularity theorem in a Finsler spacetime, Classical Quantum Gravity 33, 025003 (2016)

[2] J.A. Aledo, A. Romero, R.M. Rubio, The existence and uniqueness of standard static splitting, Classical Quantum Gravity 32, 105004 (2015)

[3] M. Abramowicz, B. Carter, J. Lasota, Optical reference geometry for stationary and static dynamics, Gen. Relativity Gravitation 20, 1173-1183 (1988)

[4] P.L. Antonelli, R.S. Ingarden, M. Matsumoto, The Theory of Sprays and Finsler Spaces with Applications in Physics and Biology, Fundamental Theories of Physics, Kluwer Academic Publishers Group, Dordrecht, 1993

[5] D. Bao, S.-S. Chern, Z. Shen, An Introduction to Riemann-Finsler Geometry, Graduate Text in Mathematics 200, Springer-Verlag, New York, 2000 
[6] E. Barletta, S. Dragomir, Gravity as a Finslerian metric phenomenon, Found. Physics 42, 436-453 (2012)

[7] J.K. Beem, Indefinite Finsler spaces and timelike spaces, Canad. J. Math. 22, 1035-1039 (1970)

[8] J.K. Beem, P.E. Ehrlich, K. Easley, Global Lorentzian Geometry, Monographs and Texbooks in Pure and Applied Mathematics 202, Marcel Dekker, New York, 1996

[9] A.N. Bernal, M. Sánchez, On smooth Cauchy hypersurfaces and Geroch's splitting theorem, Commun. Math. Phys. 243, 461-470 (2003)

[10] A.N. Bernal, M. Sánchez, Smoothness of time functions and the metric splitting of globally hyperbolic spacetimes, Comm. Math. Phys., 257, 43-50 (2005)

[11] A.N. Bernal, M. Sánchez, Globally hyperbolic spacetimes can be defined as 'causal' instead of 'strongly causal', Classical Quantum Gravity 24, 745-749 (2007)

[12] E. Caponio, A. Germinario and M. Sánchez, Convex regions of stationary spacetimes and Randers spaces. Applications to lensing and asymptotic flatness, J. Geom. Anal., (2016), DOI: $10.1007 / \mathrm{s} 12220-015-9572-\mathrm{z}$, in press

[13] E. Caponio, M.A. Javaloyes, A. Masiello, On the energy functional on Finsler manifolds and applications to stationary spacetimes, Math. Ann. 351, 365-392 (2011)

[14] E. Caponio, M.A. Javaloyes, M. Sánchez, On the interplay between Lorentzian Causality and Finsler metrics of Randers type, Rev. Mat. Iberoamericana 27, 919-952 (2011)

[15] E. Caponio, M.A. Javaloyes, M. Sánchez, Wind Finslerian structures: from Zermelo's navigation to the causality of spacetimes, arXiv:1407.5494 [math.DG] (2015)

[16] E. Caponio, G. Stancarone, Causality properties of static Finsler spacetimes, proceedings of the "1st Workshop on the State of the Art and Challenges of Research Efforts at POLIBA, 03-05 dicembre 2014, Bari, Italy, track C2 - Research Contributions". Cangemi Editore, Roma, 21-25, 2014. Available here

[17] Z. Chang, S. Wang, Lorentz and CPT violations in Finsler spacetime, arXiv:1209.3574 [hep$\mathrm{ph}$ (2012)

[18] E. Fadell, S. Husseini, Category of loop spaces of open subsets in Euclidean space, Nonlinear Anal. 17, 1153-1161 (1991)

[19] A. Fathi, A. Siconolfi, On smooth time functions, Math. Proc. Cambridge Philos. Soc. 152, 303-339 (2012)

[20] R. Gallego Torromé, P. Piccione, H. Vitório, On Fermat's principle for causal curves in time oriented Finsler spacetimes, J. Math. Phys. 53, 123511 (2012)

[21] G.W. Gibbons, C.M. Warnick, Universal properties of the near-horizon optical geometry, Phys. Rev. D 79, p. 064031 (2009)

[22] M. Hohmann, Extensions of Lorentzian spacetime geometry: from Finsler to Cartan and vice versa, Phys. Rev. D 87, 124034 (2013)

[23] M.A. Javaloyes, M. Sánchez, On the definition and examples of Finsler metrics, Ann. Scuola Norm. Sup. Pisa Cl. Sci. (5) 13, 813-858 (2014)

[24] M.A Javaloyes, M. Sánchez, Finsler metrics and relativistic spacetimes, Int. J. Geom. Methods Mod. Phys. 11, 1460032 (2014)

[25] V.A. Kostelecký, Riemann-Finsler geometry and Lorentz-violating kinematics, Phys. Lett. B 701, 137 (2011)

[26] V.A. Kostelecký, N. Russell, R. Tso, Bipartite Riemann-Finsler geometry and Lorentz violation, Phys. Lett. B 716, 470 (2012)

[27] A.P. Kouretsis, M. Stathakopoulos, P.C. Stavrinos, Covariant kinematics and gravitational bounce in Finsler space-times, Phys. Rev. D 86, 124025 (2012)

[28] C. Lämmerzahl, V. Perlick, W. Hasse, Observable effects in a class of spherically symmetric static Finsler spacetimes, Phys. Review D 86, 104042 (2012)

[29] X. Li, Z. Chang, Exact solution of vacuum field equation in Finsler spacetime, Phys. Rev. D 90, 064049 (2014)

[30] R.L. Lovas, On the Killing vector fields of generalized metrics, SUT J. Math. 40, 133-156 (2004)

[31] A. Masiello, Variational Methods in Lorentzian Geometry. Pitman Research Notes in Mathematics Series 309, Longman, London, 1994

[32] V.S. Matveev, Can we make a Finsler metric complete by a trivial projective change? Recent trends in Lorentzian geometry, Springer Proc. Math. Stat. bf 26. 231-242, Springer, New York, 2013 
[33] T. Mestdag, J.Szilasi, V. Tóth, On the geometry of generalized metrics, Publ. Math. Debrecen 62, 511-545 (2003)

[34] E. Minguzzi, Light cones in Finsler spacetime, Comm. Math. Phys. 334, 1529-1551 (2015)

[35] E. Minguzzi, Convex neighborhoods for Lipschitz connections and sprays, Monatsh. Math. $\mathbf{1 7 7}, 569-625(2015)$

[36] B. O'Neill, Semi-Riemannian Geometry, Pure and Applied Mathematics 103, Academic Press, Inc., New York, 1983

[37] V. Perlick, Gravitational lensing from a spacetime perspective, Living Reviews in Relativity, http://www.livingreviews.org/lrr-2004-9 (2004)

[38] V. Perlick, Fermat principle in Finsler spacetimes, Gen. Relativity Gravitation 38, 365-380 (2006)

[39] C. Pfeifer, M.N.R. Wohlfarth, Causal structure and electrodynamics on Finsler spacetimes, Phys. Rev. D 84, 044039 (2011)

[40] C. Pfeifer, M.N.R. Wohlfarth, Finsler geometric extension of Einstein gravity, Phys. Rev. D 85, 064009 (2012)

[41] H.-B. Rademacher, A sphere theorem for non-reversible Finsler metrics, Math. Ann. 328, 373-387 (2004)

[42] S. Rajpoot, S. Vacaru, Black ring and Kerr ellipsoid - solitonic configurations in modified Finsler gravity, Int. J. Geom. Meth. Mod. Phys. 12, 1550102 (2015)

[43] N. Russell, Remarks on Finsler geometry and Lorentz violation, arXiv:1308.3442 [hep-ph] (2013)

[44] M. Sánchez, J.M.M. Senovilla, A note on the uniqueness of global static decompositions, Classical Quantum Gravity 24, 6121-6126 (2007)

[45] M. Schreck, Classical kinematics and Finsler structures for nonminimal Lorentz-violating fermions, The European Physical Journal C, 75:187 (2015)

[46] Z. Shen, Lectures on Finsler geometry, World Scientific Publishing Co., Singapore, 2001

[47] J.E.G Silva, C.A.S. Almeida, Kinematics and dynamics in a bipartite-Finsler spacetime, Phys. Lett. B 731, 74-79 (2014)

[48] P. Stavrinos, S. Vacaru, Cyclic and ekpyrotic universes in modified Finsler osculating gravity on tangent Lorentz bundles, Classical Quantum Gravity 30, 055012 (2013)

[49] S. Vacaru, Principles of Einstein-Finsler gravity and perspectives in modern cosmology, Int. J. Mod. Phys. D 21, 1250072 (2012)

[50] S. Vacaru, Modified dispersion relations in Horava-Lifshitz gravity and Finsler brane models Gen. Relativity Gravitation 44, 1015-1042 (2012)

Dipartimento di Meccanica, Matematica e Management,

Politecnico di Bari, Via Orabona 4, 70125, Bari, Italy

E-mail address: caponio@poliba.it

Dipartimento di Matematica

Università degli Studi di Bari, Via Orabona 4, 70125 Bari, Italy

E-mail address: giuseppe.stancarone@uniba.it 